\title{
Global Existence and Decay Estimates for Quasilinear Wave Equations with Nonuniform Dissipative Term
}

\author{
By
}

Tomonari WatANABE

(Hiroshima University, Japan)

\begin{abstract}
In this paper, we study a Cauchy problem for quasilinear wave equations with dissipative term in Sobolev space $H^{L} \times H^{L-1}(L \geq[d / 2]+3)$. The coefficients of the dissipative term depends on space variables and may vanish in some compact region. In order to control the derivatives of the dissipative coefficients, we introduce a rescaling argument. Using the argument, we obtain a global existence theorem and decay estimates with additional assumptions for the initial data.

Key Words and Phrases. Dissipative quasilinear wave equations, Space variable coefficients, Time decay estimates.

2010 Mathematics Subject Classification Numbers. 35L72, 35L15.
\end{abstract}

\section{Introduction}

In this paper, we consider the following Cauchy problem for quasilinear wave equations with nonuniform dissipative term in $\boldsymbol{R}^{d}(d \geq 1)$ :

$$
\text { (DW) } \begin{cases}\left(\partial_{t}^{2}-\triangle+B(x) \partial_{t}\right) u(t, x)=N[u, u](t, x), & (t, x) \in[0, \infty) \times \boldsymbol{R}^{d}, \\ u(0, x)=u_{0}(x), \partial_{t} u(0, x)=u_{1}(x), & x \in \boldsymbol{R}^{d},\end{cases}
$$

where $u=\left(u^{1}, u^{2}, \ldots, u^{d}\right)$ is a vector valued function, $\triangle u(t, x)=\left(\triangle u^{1}, \triangle u^{2}, \ldots\right.$, $\left.\triangle u^{d}\right), \partial_{t} u=\left(\partial_{t} u^{1}, \partial_{t} u^{2}, \ldots, \partial_{t} u^{d}\right)$ and $\partial_{t}^{2} u=\left(\partial_{t}^{2} u^{1}, \partial_{t}^{2} u^{2}, \ldots, \partial_{t}^{2} u^{d}\right)$. The initial data $\left(u_{0}, u_{1}\right)$ in (DW) belong to $H^{L} \times H^{L-1}$, where $H^{L}$ is the Sobolev space in $\boldsymbol{R}^{d}$. The coefficient function $B(x)$ is $d \times d$ symmetric matrix-valued function and quasilinear term $N[u, v](t, x)$ is defined by

$$
N[u, v]=\left(N[u, v]^{i}\right)_{i=1,2, \ldots, d}=\left(\sum_{j, k, l, m, n=1}^{d} N_{l m n}^{i j k} \partial_{l}\left(\partial_{m} u^{j} \partial_{n} v^{k}\right)\right)_{i=1,2, \ldots, d} .
$$

For $B$ and $N$, we make the following assumptions:

(B0) $B(x)=\left(B_{p q}(x)\right)_{p, q=1,2, \ldots, d}$ is $d \times d$ symmetric matrix-valued function whose components belong to $\mathscr{B}^{\infty}$, where $\mathscr{B}^{\infty}$ is a function space of smooth functions with bounded derivatives. 
(B1) $B(x)$ is nonnegative, i.e.

$$
\sum_{p, q=1}^{d} B_{p q}(x) \eta_{p} \eta_{q} \geq 0 \quad\left(\eta, x \in \boldsymbol{R}^{d}\right) .
$$

(B2) There exist $b_{0}>0$ and $R>0$ such that

$$
\sum_{p, q=1}^{d} B_{p q}(x) \eta_{p} \eta_{q} \geq b_{0}|\eta|^{2} \quad\left(|x| \geq R, \eta \in \boldsymbol{R}^{d}\right) .
$$

(N0) $N_{l m n}^{i j k} \in \boldsymbol{R}(i, j, k, l, m, n=1,2, \ldots, d)$.

(N1) $N_{l m n}^{i j k}=N_{m l n}^{j i k}=N_{l n m}^{i k j}(i, j, k, l, m, n=1,2, \ldots, d)$.

If we assume $B=$ const $>0$, there are many results (see [3], [7], [9], [10] etc.). For general quasilinear version including $N$, Racke [13] shows that there exists a unique global solution and decay estimates when the initial data are sufficiently smooth and small. On the other hand, the global existence is not obvious when $B$ is not positive constant. Indeed if $B$ vanishes, (DW) becomes the quasilinear wave equations. Then it is well known that no matter how small the initial data, there does not exist globally defined smooth solution in general (e.g. [6]). However if the nonlinear term $N$ has "Null Condition", then (DW) has a global smooth solution for sufficiently smooth and small initial data (e.g. [1], [8], [14]).

There are some results for nonuniform dissipative term which satisfies (B1)-(B2) ([2], [5], [11], [12] etc.). In particular, Nakao [12] obtains a global existence when the nonlinear term have second order derivatives. He assume that the order of the nonlinear term $p$ satisfies $p \geq 3$. In this paper, we treat the case of $p=2$, i.e. the quasilinear term $N[u, u]$ is a quadratic function. In order to treat the quadratic case, we introduce a rescaling argument and energy norms for controlling higher order derivatives. There norms are used for the nonlinear wave equations.

We prove the following global existence theorem:

Theorem 1.1. Let $L \geq L_{0}=[d / 2]+3$. Then there exists a small constant $\hat{\delta}>0$ such that if the initial data $\left(u_{0}, u_{1}\right) \in H^{L} \times H^{L-1}$ satisfies

$$
\left\|u_{0}\right\|_{H^{L_{0}}}^{2}+\left\|u_{1}\right\|_{H^{L_{0}-1}}^{2} \leq \hat{\delta}
$$

then there exists a unique solution to (DW) in $\bigcap_{j=0}^{L} C^{j}\left([0, \infty) ; H^{L-j}\right)$.

We note that $L \geq L_{0}=[d / 2]+3$ is reasonable. Because we use the assumption to obtain a local solution in usual sense (see [10], [12], [16]). Furthermore the assumption is necessary to estimate the quasilinear term for the 
purpose of extending the solution. Indeed using product estimate (proposition 2.2) and embedding (proposition 2.1), we calculate as

$$
\begin{aligned}
\left\|\partial_{l}\left(\partial_{m} u \partial_{n} u\right)\right\|_{H^{L-2}} & \leq C\left\{\|\nabla u\|_{H^{L-2}}\left\|\nabla^{2} u\right\|_{\infty}+\|\nabla u\|_{\infty}\left\|\nabla^{2} u\right\|_{H^{L-2}}\right\} \\
& \leq C\|\nabla u\|_{H^{L-1}}\|\nabla u\|_{H^{L_{0}-1}} \leq C\|\nabla u\|_{H^{L-1}}^{2}
\end{aligned}
$$

(see lemma 5.1).

We also remark that the smallness of $\left\|u_{0}\right\|_{L^{2}}$ is needed to estimate the higher order derivatives of nonuniform dissipative term as $\nabla^{\alpha}(B(x) u)$ (see lemma 5.2). Indeed if $B=$ Const $>0$, we can prove theorem 1.1 under the assumption $\left\|\nabla u_{0}\right\|_{H^{L_{0}-1}}^{2}+\left\|u_{1}\right\|_{H^{L_{0}-1}}^{2} \leq \hat{\delta}$ instead of (1).

We show the decay estimates with the additional assumptions as follow:

Theorem 1.2. In addition to the assumptions in theorem 1.1, we assume that one of the following (H1)-(H3) is held:

(H1) $d \geq 3$ and there exists $1 \leq p \leq 2 d /(d+2)$ such that $B u_{0}+u_{1} \in L^{p}$,

(H2) $d \geq 3$ and $|\cdot|\left\{B u_{0}+u_{1}\right\} \in L^{2}$,

(H3) $d=1$ or $2,|\cdot|\left\{B u_{0}+u_{1}\right\} \in L^{1}$ and

$$
\int_{\boldsymbol{R}^{d}} B(x) u_{0}(x)+u_{1}(x) d x=0 .
$$

Then for any $\mu\left(0 \leq \mu \leq L-L_{0}\right)$, there exists a constant $E_{0}>0$ depending on $\left(u_{0}, u_{1}\right)$ such that the global solution $u$ to $(\mathrm{DW})$ satisfies following estimates:

$$
\begin{gathered}
\left\|\partial_{t}^{\mu} u(t)\right\|_{H^{L-\mu}}^{2}+\left\|\partial_{t}^{\mu+1} u(t)\right\|_{H^{L-\mu-1}}^{2} \leq E_{0}(1+t)^{-2 \mu-1}, \\
\left\|\nabla \partial_{t}^{\mu} u(t)\right\|_{2}^{2}+\left\|\partial_{t}^{\mu+1} u(t)\right\|_{2}^{2} \leq E_{0}(1+t)^{-2 \mu-2}, \\
\left\|\partial_{t}^{\mu} u(t)\right\|_{\infty}^{2} \leq E_{0}(1+t)^{-2 \mu-1} .
\end{gathered}
$$

Furthermore if $L>L_{0}$, it holds that

$$
\|\Delta u(t)\|_{2}^{2} \leq E_{0}(1+t)^{-3}
$$

Ikehata [5] treats the linear or semilinear cases and obtains the result corresponding to theorem 1.2 with the assumption (H2). Thus theorem 1.2 means that even for the quasilinear case, the same type decay properties as in [5] are held. For the proof, we use the method which is introduced in [5]. However, to estimate the quasilinear term, we use the time weighted higher order estimates (see $\S 4$ ).

In this paper, the rescaling argument plays an important role (for the definition of the scaling, see $\S 2$ ). We have to control terms include $\nabla^{\beta} B(x)$ to calculate the higher order energy estimates. Using a usual way, we need the smallness of $\left\|\nabla^{\beta} B(x)\right\|_{\infty}$ or delicate calculation. However, using the rescaling, 
we can control the smallness of $\left\|\nabla^{\beta} B(x)\right\|_{\infty}$ by a scale parameter. Therefore we can expect to prove the higher order energy estimates with simple argument.

\section{Preliminaries}

Throughout this paper, $\|\cdot\|_{p}$ and $\|\cdot\|_{H^{l}}$ stand for the usual $L^{p}\left(\boldsymbol{R}^{d}\right)$-norm and $H^{l}\left(\boldsymbol{R}^{d}\right)$-norm. Furthermore, we adopt

$$
\langle g, f\rangle=\sum_{i=1}^{d} \int_{\boldsymbol{R}^{d}} f^{i}(x) g^{i}(x) d x
$$

as the usual $L^{2}\left(\boldsymbol{R}^{d}\right)$-inner product.

We consider the rescaling to (DW). Let $u$ be the solution to (DW). We define $v(t, x)=\lambda^{-1} u(\lambda t, \lambda x)(\lambda>0)$, then $v$ satisfies

$$
\begin{aligned}
\partial_{t}^{2} v(t, x)-\Delta v(t, x) & =\lambda\left\{\partial_{t}^{2} u(\lambda t, \lambda x)-\Delta u(\lambda t, \lambda x)\right\} \\
& =-\lambda B(\lambda x) \partial_{t} u(\lambda t, \lambda x)+\lambda N[u, u](\lambda t, \lambda x) \\
& =-\lambda B(\lambda x) \partial_{t} v(t, x)+N[v, v](t, x) .
\end{aligned}
$$

Thus $v$ is the solution to the following Cauchy problem (DW) $\lambda$ :

$$
(\mathrm{DW})_{\lambda} \begin{cases}\left(\partial_{t}^{2}-\triangle+B_{\lambda}(x) \partial_{t}\right) v(t, x)=N[v, v](t, x), & (t, x) \in[0, \infty) \times \boldsymbol{R}^{d}, \\ v(0, x)=v_{0}(x), \partial_{t} v(0, x)=v_{1}(x), & x \in \boldsymbol{R}^{d},\end{cases}
$$

where $B_{\lambda}(x)=\lambda B(\lambda x), v_{0}(x)=\lambda^{-1} u_{0}(\lambda x), v_{1}(x)=u_{1}(\lambda x)$. Now $B_{\lambda}$ satisfies

(B1) ${ }_{\lambda} \quad B_{\lambda}(x)$ is nonnegative.

(B2) $\lambda$ There exist $b_{0}>0$ and $R>0$ such that

$$
\sum_{p, q=1}^{d}\left(B_{\lambda}\right)_{p q}(x) \eta_{p} \eta_{q}=\sum_{p, q=1}^{d} \lambda B_{p q}(\lambda x) \eta_{p} \eta_{q} \geq \lambda b_{0}|\eta|^{2} \quad\left(|x| \geq \frac{R}{\lambda}, \eta \in \boldsymbol{R}^{d}\right)
$$

instead of (B1) and (B2). Furthermore $B_{\lambda}$ satisfies

(B3) $\lambda \quad\left\|\nabla^{b} B_{\lambda}\right\|_{\infty} \leq \lambda^{|b|+1}\left\|\nabla^{b} B\right\|_{\infty}$.

We consider $(\mathrm{DW})_{\lambda}$ for $\lambda \leq 1$ instead of $(\mathrm{DW})$.

We introduce the known results. First, we use the following two lemmas for estimating the nonlinearity terms. For the proof, see for example [16] section 13.

Lemma 2.1. There exists a constant $C>0$ such that

$$
\|f\|_{\infty} \leq C\|f\|_{H^{[d / 2]+1}} .
$$


Lemma 2.2. Let $k \in \boldsymbol{Z}$ and $b, c \in \boldsymbol{Z}_{+}^{d}$ satisfy $|b|+|c|=k$. There exists $C>0$ such that

$$
\begin{aligned}
\left\|\nabla^{b} f \nabla^{c} g\right\|_{2} & \leq C\|f\|_{\infty}\left\|\nabla^{b+c} g\right\|_{2}+C\left\|\nabla^{b+c} f\right\|_{2}\|g\|_{\infty} \\
& \leq C\|f\|_{\infty}\|g\|_{H^{k}}+C\|f\|_{H^{k}}\|g\|_{\infty} .
\end{aligned}
$$

Next, we prepare the Poincare type inequality of $B_{\lambda}$ for the proof of global existence.

Lemma 2.3 (Poincare type inequality). There exists a constant $C_{1} \geq 1 / 4$ such that

$$
\|f\|_{2}^{2} \leq \frac{C_{1}}{\lambda}\left\langle f, B_{\lambda} f\right\rangle+\frac{C_{1}}{\lambda^{2}}\|\nabla f\|_{2}^{2} \quad(\lambda>0) .
$$

Proof. We define $U_{r}=\left\{x \in \boldsymbol{R}^{d}|| x \mid \leq r\right\}$. Using Poincare inequality (see [4]), we obtain the following estimate:

$$
\int_{U_{r}}|f(x)|^{2} d x \leq r^{2} \int_{U_{r}}|\nabla f(x)|^{2} d x .
$$

Let $\rho \in C_{0}^{\infty}\left(\boldsymbol{R}^{d}\right)$ be a function satisfying $0 \leq \rho \leq 1, \rho(x)=1 \quad(|x| \leq 1), \rho(x)=0$ $(|x| \geq 3 / 2)$. For any $f \in H^{1}\left(\boldsymbol{R}^{d}\right)$ and $\lambda>0$ we define $\rho_{\lambda}(x)=\rho(\lambda x / R)$, then because of $\rho_{\lambda} f \in H_{0}^{1}\left(U_{2 R / \lambda}\right)$ we have

$$
\begin{aligned}
\|f\|_{2}^{2}= & \int_{\boldsymbol{R}^{d}}\left|\rho_{\lambda}(x) f(x)\right|^{2} d x+\left.\int_{\boldsymbol{R}^{d}}\left(1-\left|\rho_{\lambda}(x)\right|^{2}\right) f(x)\right|^{2} d x \\
= & \int_{|x| \leq 2 R / \lambda}\left|\rho_{\lambda}(x) f(x)\right|^{2} d x+\left.\int_{|x| \geq R / \lambda}\left(1-\left|\rho_{\lambda}(x)\right|^{2}\right) f(x)\right|^{2} d x \\
\leq & \frac{4 R^{2}}{\lambda^{2}} \int_{|x| \leq 2 R / \lambda}\left|\nabla\left(\rho_{\lambda}(x) f(x)\right)\right|^{2} d x+\int_{|x| \geq R / \lambda}|f(x)|^{2} d x \\
\leq & \frac{4 R^{2}}{\lambda^{2}} \int_{R / \lambda \leq|x| \leq 2 R / \lambda}\left|\nabla \rho_{\lambda}(x)\right|^{2}|f(x)|^{2} d x \\
& +\frac{4 R^{2}}{\lambda^{2}} \int_{|x| \leq 2 R / \lambda}\left|\rho_{\lambda}(x)\right|^{2}|\nabla f(x)|^{2} d x+\int_{|x| \geq R / \lambda}|f(x)|^{2} d x \\
\leq & \left(4 R^{2}\|\nabla \rho\|_{\infty}^{2}+1\right) \int_{|x| \geq R / \lambda}|f(x)|^{2} d x+\frac{4 R^{2}}{\lambda^{2}} \int_{R^{d}}|\nabla f(x)|^{2} d x \\
\leq & \frac{4 R^{2}\|\nabla \rho\|_{\infty}^{2}+1}{b_{0} \lambda}\left\langle f, B_{\lambda} f\right\rangle+\frac{4 R^{2}}{\lambda^{2}}\|\nabla f\|_{2}^{2} .
\end{aligned}
$$

Hence we get (6). 
Finally, we introduce Hardy inequality and Gagliardo-Nirenberg inequality. For the proof, see for example [15] appendix A.

Lemma 2.4. Let $d \geq 3$. There exists a constant $C>0$ such that any $f \in H^{1}$ satisfies

$$
\left\|\frac{f}{|\cdot|}\right\|_{2} \leq C\|\nabla f\|_{2}
$$

Lemma 2.5. Assume $1 \leq q<d$ and $1 / p=1 / q-1 / d$. Then there exists a constant $C>0$ depend on $p, q, d$ such that

$$
\|g\|_{p} \leq C\|\nabla g\|_{q}
$$

\section{Global existence}

In this section we prove theorem 1.1. First we define some notations. For any $h, g: \boldsymbol{R}^{d} \rightarrow \boldsymbol{R}^{d}$, we define $[h ; \nabla g]: \boldsymbol{R}^{d} \rightarrow \boldsymbol{R}^{d}$ as follows:

$$
([h ; \nabla g])^{i}(x)=h(x) \cdot \nabla g^{i}(x) \quad(i=1,2, \ldots, d) .
$$

The energy $E(u(t))$ and higher order energies $E_{\bar{L}}(u(t))$ of $u$ are defined by

$$
E(u(t))=\frac{1}{2}\left\{\left\|\partial_{t} u(t)\right\|_{2}^{2}+\|\nabla u(t)\|_{2}^{2}\right\}
$$

and

$$
E_{\bar{L}}(u(t))=\sum_{|a| \leq \bar{L}-1} E\left(\nabla^{a} u(t)\right) .
$$

Moreover we define

$$
\tilde{N}[u, v, w](t)=\sum_{i, j, k, l, m, n=1}^{d} N_{l m n}^{i j k} \int_{\boldsymbol{R}^{d}} \partial_{l} u^{i}(t, x) \partial_{m} v^{j}(t, x) \partial_{n} w^{k}(t, x) d x
$$

and

$$
\tilde{E}_{\bar{L}, \mu}(u(t))=E_{\bar{L}-\mu}\left(\partial_{t}^{\mu} u(t)\right)+\sum_{|a| \leq \bar{L}-\mu-1} \tilde{N}\left[\partial_{t}^{\mu} \nabla^{a} u, \partial_{t}^{\mu} \nabla^{a} u, u\right](t) .
$$

The function spaces $X_{\delta, T}$ and $X_{\delta}$ are defined by

$$
X_{\delta, T}=\left\{u \in \bigcap_{j=0}^{L} C^{j}\left([0, T] ; H^{L-j}\right) \mid E_{L_{0}}(u(t)) \leq \delta^{2} \quad(0 \leq t \leq T)\right\}
$$

and

$$
X_{\delta}=\left\{u \in \bigcap_{j=0}^{L} C^{j}\left([0, \infty) ; H^{L-j}\right) \mid E_{L_{0}}(u(t)) \leq \delta^{2} \quad(0 \leq t<\infty)\right\} .
$$


Let $L_{0} \leq \bar{L} \leq L, \mu \leq L-L_{0}$ and $\lambda>0$, we define $G_{\bar{L}, \mu}(v(t))$ below.

$$
\begin{aligned}
G_{\bar{L}, \mu}(v(t))= & \frac{C_{0}}{\lambda} \tilde{E}_{\bar{L}, \mu}(v(t))+\frac{b_{0}(2 d-1)}{4} \sum_{|a| \leq \bar{L}-\mu-1}\left\langle\partial_{t}^{\mu} \nabla^{a} v(t), \partial_{t}^{\mu+1} \nabla^{a} v(t)\right\rangle \\
& +\frac{b_{0}(2 d-1)}{8} \sum_{|a| \leq \bar{L}-\mu-1}\left\langle\partial_{t}^{\mu} \nabla^{a} v(t), B_{\lambda} \partial_{t}^{\mu} \nabla^{a} v(t)\right\rangle \\
& +\sum_{|a| \leq \bar{L}-\mu-1}\left\langle\partial_{t}^{\mu+1} \nabla^{a} v(t),\left[h ; \nabla \partial_{t}^{\mu} \nabla^{a} v\right](t)\right\rangle,
\end{aligned}
$$

where

$$
C_{0}=\max \left\{\left(b_{0} R d^{2}+\frac{C_{1} b_{0}(2 d-1)}{2}\right) \times 4, d, 2\|B\|_{\infty} b_{0}^{2} R^{2} \times \frac{8}{b_{0}}\right\},
$$

and

$$
\phi(r)=\left\{\begin{array}{ll}
b_{0}, & (r \leq R / \lambda) \\
b_{0} R /(\lambda r), & (r \geq R / \lambda)
\end{array}, \quad h(x)=x \phi(|x|) .\right.
$$

We need the energy estimate to prove the global existence.

Lemma 3.1. Let $L_{0} \leq \bar{L} \leq L$ and $\mu \leq \bar{L}-L_{0}$. There exists a constant $C>0$ such that for any $\delta, \lambda, T>0$ and a local solution $v \in X_{\delta, T}$ to $(\mathrm{DW})_{\lambda}$ satisfy

$$
\begin{aligned}
& \frac{d}{d t} G_{\bar{L}, \mu}(v(t))+\frac{b_{0}}{2} E_{\bar{L}-\mu}\left(\partial_{t}^{\mu} v(t)\right) \\
& \quad \leq \lambda C E_{\bar{L}-\mu}\left(\partial_{t}^{\mu} v(t)\right)+\frac{C}{\lambda} D_{\bar{L}, \mu}(v(t))+\frac{2\|B\|_{\infty} b_{0}^{2} R^{2}}{C_{0}} E_{\bar{L}-\mu}\left(\partial_{t}^{\mu} v(t)\right),
\end{aligned}
$$

where $C_{0}>0$ is the constant give in (16), and

$$
D_{\bar{L}, \mu}(v(t))=E_{\bar{L}-\mu}^{1 / 2}\left(\partial_{t}^{\mu} v(t)\right) \cdot\left(\sum_{v=0}^{\mu} E_{L_{0}}^{1 / 2}\left(\partial_{t}^{\mu-v} v(t)\right) E_{\bar{L}-v}^{1 / 2}\left(\partial_{t}^{v} v(t)\right)\right) .
$$

The proof of the lemma 3.1 is given in section 5. In what follows, assuming lemma 3.1 , we derive the energy estimates for $(\mathrm{DW})_{\lambda}$.

\subsection{Energy estimate}

We prove the higher order energy estimates of $(\mathrm{DW})_{\lambda}$.

Lemma 3.2. Let $L_{0} \leq \bar{L} \leq L$ and $\mu \leq \bar{L}-L_{0}$. There exists $C>0$ such that if $\delta, \lambda>0$ are sufficiently small, then the local solution $v \in X_{\delta, T}$ to $(\mathrm{DW})_{\lambda}$ satisfies 
(20) $\frac{1}{C}\left\{\lambda\left\|\partial_{t}^{\mu} v(t)\right\|_{2}^{2}+\frac{1}{\lambda} E_{\bar{L}-\mu}\left(\partial_{t}^{\mu} v(t)\right)\right\} \leq G_{\bar{L}, \mu}\left(\partial_{t}^{\mu} v(t)\right)$

$$
\leq C\left\{\lambda\left\|\partial_{t}^{\mu} v(t)\right\|_{2}^{2}+\frac{1}{\lambda} E_{\bar{L}-\mu}\left(\partial_{t}^{\mu} v(t)\right)\right\} .
$$

Proof. Let $v \in X_{\delta, T}$. First it holds that

$$
\frac{1}{2} E_{\bar{L}-\mu}\left(\partial_{t}^{\mu} v(t)\right) \leq \tilde{E}_{\bar{L}, \mu}(v(t)) \leq \frac{3}{2} E_{\bar{L}-\mu}\left(\partial_{t}^{\mu} v(t)\right)
$$

Because

$$
\begin{aligned}
\left|\sum_{|a| \leq \bar{L}-\mu-1} \tilde{N}\left[\partial_{t}^{\mu} \nabla^{a} v, \partial_{t}^{\mu} \nabla^{a} v, v\right](t)\right| & \leq C \sum_{|a| \leq \bar{L}-\mu-1}\|\nabla v(t)\|_{\infty}\left\|\nabla \partial_{t}^{\mu} \nabla^{a} v(t)\right\|_{2}^{2} \\
& \leq C \sum_{|a| \leq \bar{L}-\mu-1}\|\nabla v(t)\|_{H^{[d / 2]+1}}\left\|\nabla \partial_{t}^{\mu} \nabla^{a} v(t)\right\|_{2}^{2} \\
& \leq \delta C E_{\bar{L}-\mu}\left(\partial_{t}^{\mu} v(t)\right),
\end{aligned}
$$

we choose $\delta$ sufficiently small depend on $d$ and $N$, then we get (21). It follows from lemma 2.3 that

$$
\begin{gathered}
\left|\sum_{|a| \leq \bar{L}-\mu-1}\left\langle\partial_{t}^{\mu} \nabla^{a} v(t), \partial_{t}^{\mu+1} \nabla^{a} v(t)\right\rangle\right| \\
\leq \sum_{|a| \leq \bar{L}-\mu-1}\left\{\frac{\lambda}{4 C_{1}}\left\|\partial_{t}^{\mu} \nabla^{a} v(t)\right\|_{2}^{2}+\frac{C_{1}}{\lambda}\left\|\partial_{t}^{\mu+1} \nabla^{a} v(t)\right\|_{2}^{2}\right\} \\
\leq \sum_{|a| \leq \bar{L}-\mu-1}\left\{\frac{1}{4}\left\langle\partial_{t}^{\mu} \nabla^{a} v(t), B_{\lambda} \partial_{t}^{\mu} \nabla^{a} v(t)\right\rangle\right. \\
\left.\quad+\frac{1}{4 \lambda}\left\|\nabla \partial_{t}^{\mu} \nabla^{a} v(t)\right\|_{2}^{2}+\frac{C_{1}}{\lambda}\left\|\partial_{t}^{\mu+1} \nabla^{a} v(t)\right\|_{2}^{2}\right\} \\
\leq \frac{1}{4} \sum_{|a| \leq \bar{L}-\mu-1}\left\langle\partial_{t}^{\mu} \nabla^{a} v(t), B_{\lambda} \partial_{t}^{\mu} \nabla^{a} v(t)\right\rangle+\frac{2 C_{1}}{\lambda} E_{\bar{L}-\mu}\left(\partial_{t}^{\mu} v(t)\right) .
\end{gathered}
$$

Using

$$
\|h\|_{\infty} \leq \frac{b_{0} R}{\lambda} \quad \text { and } \quad\|\nabla h\|_{\infty} \leq 2 b_{0}
$$


we get

$$
\begin{aligned}
& \left|\sum_{|a| \leq \bar{L}-\mu-1}\left\langle\partial_{t}^{\mu+1} \nabla^{a} v(t),\left[h ; \nabla \partial_{t}^{\mu} \nabla^{a} v\right](t)\right\rangle\right| \\
& \quad \leq \sum_{|a| \leq \bar{L}-\mu-1} \sum_{k, j=1}^{d}\left|\int_{R^{d}} \partial_{t}^{\mu+1} \nabla^{a} v^{k}(t) \partial_{j} \partial_{t}^{\mu} \nabla^{a} v^{k}(t) h^{j} d x\right| \\
& \quad \leq \sum_{|a| \leq \bar{L}-\mu-1} \sum_{k, j=1}^{d}\left\|\partial_{t}^{\mu+1} \nabla^{a} v^{k}(t)\right\|_{2}\left\|\partial_{j} \partial_{t}^{\mu} \nabla^{a} v^{k}(t)\right\|_{2}\left\|h^{j}\right\|_{\infty} \\
& \quad \leq d^{2} \sum_{|a| \leq \bar{L}-\mu-1}\left\{\frac{1}{2}\left\|\partial_{t}^{\mu+1} \nabla^{a} v(t)\right\|_{2}^{2}+\frac{1}{2}\left\|\nabla \partial_{t}^{\mu} \nabla^{a} v(t)\right\|_{2}^{2}\right\}\|h\|_{\infty} \\
& \leq \frac{b_{0} R d^{2}}{\lambda} E_{\bar{L}-\mu}\left(\partial_{t}^{\mu} v(t)\right) .
\end{aligned}
$$

Using (21), (22), (24) and (16) we have

$$
\begin{aligned}
G_{\bar{L}, \mu}(v(t)) \geq & \frac{1}{\lambda}\left(\frac{C_{0}}{2}-b_{0} R d^{2}-\frac{C_{1} b_{0}(2 d-1)}{2}\right) E_{\bar{L}-\mu}\left(\partial_{t}^{\mu} v(t)\right) \\
& +\frac{b_{0}(2 d-1)}{16} \sum_{|a| \leq \bar{L}-\mu-1}\left\langle\partial_{t}^{\mu} \nabla^{a} v(t), B_{\lambda} \partial_{t}^{\mu} \nabla^{a} v(t)\right\rangle \\
\geq & \frac{1}{\lambda}\left(b_{0} R d^{2}+\frac{C_{1} b_{0}(2 d-1)}{2}\right) E_{\bar{L}-\mu}\left(\partial_{t}^{\mu} v(t)\right) \\
& +\frac{b_{0}(2 d-1)}{16} \sum_{|a| \leq \bar{L}-\mu-1}\left\langle\partial_{t}^{\mu} \nabla^{a} v(t), B_{\lambda} \partial_{t}^{\mu} \nabla^{a} v(t)\right\rangle .
\end{aligned}
$$

So there exists a constant $C$ such that $v$ satisfies

$$
\frac{1}{\lambda} E_{\bar{L}-\mu}\left(\partial_{t}^{\mu} v(t)\right)+\sum_{|a| \leq \bar{L}-\mu-1}\left\langle\partial_{t}^{\mu} \nabla^{a} v(t), B_{\lambda} \partial_{t}^{\mu} \nabla^{a} v(t)\right\rangle \leq C G_{\bar{L}, \mu}(v(t)) .
$$

Furthermore using (25) and lemma 2.3, we have

$$
\begin{aligned}
\left\|\partial_{t}^{\mu} v(t)\right\|_{2}^{2} & \leq \frac{C_{1}}{\lambda}\left\langle B_{\lambda} \partial_{t}^{\mu} v(t), \partial_{t}^{\mu} v(t)\right\rangle+\frac{C_{1}}{\lambda^{2}}\left\|\nabla \partial_{t}^{\mu} v\right\|_{2}^{2} \\
& \leq \frac{C_{1}}{\lambda} \sum_{|a| \leq \bar{L}-\mu-1}\left\langle B_{\lambda} \partial_{t}^{\mu} \nabla^{a} v(t), \partial_{t}^{\mu} \nabla^{a} v(t)\right\rangle+\frac{2 C_{1}}{\lambda^{2}} E_{\bar{L}-\mu}\left(\partial_{t}^{\mu} v(t)\right)
\end{aligned}
$$




$$
\begin{aligned}
& \leq \frac{2 C_{1}}{\lambda}\left(\sum_{|a| \leq \bar{L}-\mu-1}\left\langle B_{\lambda} \partial_{t}^{\mu} \nabla^{a} v(t), \partial_{t}^{\mu} \nabla^{a} v(t)\right\rangle+\frac{1}{\lambda} E_{\bar{L}-\mu}\left(\partial_{t}^{\mu} v(t)\right)\right) \\
& \leq \frac{2 C_{1} C}{\lambda} G_{\bar{L}, \mu}(v(t)) .
\end{aligned}
$$

From (25) and (26), there exists a constant $C>0$ such that $v$ satisfies

$$
\frac{1}{C}\left\{\lambda\left\|\partial_{t}^{\mu} v(t)\right\|_{2}^{2}+\frac{1}{\lambda} E_{\bar{L}-\mu}\left(\partial_{t}^{\mu} v(t)\right)\right\} \leq G_{\bar{L}, \mu}(v(t)) .
$$

On the other hand using (21), (22) and (24), we get

$$
\begin{aligned}
G_{\bar{L}, \mu}(v(t)) \leq & \frac{1}{\lambda}\left(\frac{3 C_{0}}{2 \lambda}+\frac{C_{1} b_{0}(2 d-1)}{2}+b_{0} R d^{2}\right) E_{\bar{L}-\mu}\left(\partial_{t}^{\mu} v(t)\right) \\
& +\frac{3 b_{0}(2 d-1)}{16} \sum_{|a| \leq \bar{L}-\mu-1}\left\langle\partial_{t}^{\mu} \nabla^{a} v(t), B_{\lambda} \partial_{t}^{\mu} \nabla^{a} v(t)\right\rangle \\
\leq & \frac{C}{\lambda} E_{\bar{L}-\mu}\left(\partial_{t}^{\mu} v(t)\right)+C\left\|B_{\lambda}\right\|_{\infty} \sum_{|a| \leq \bar{L}-\mu-1}\left\|\partial_{t}^{\mu} \nabla^{a} v(t)\right\|_{2}^{2} \\
\leq & \frac{C}{\lambda} E_{\bar{L}-\mu}\left(\partial_{t}^{\mu} v(t)\right)+\lambda C\left\|\partial_{t}^{\mu} v(t)\right\|_{2}^{2} .
\end{aligned}
$$

So combining (27) and (28), we get (20). This completes the proof of lemma 3.2 .

Lemma 3.3. Let $L_{0} \leq \bar{L} \leq L$. There exist sufficiently small constants $\lambda$ and $\delta$ such that if $v \in X_{\delta, T}$ is a local solution to $(\mathrm{DW})_{\lambda}$ then $v$ satisfies

$$
\frac{d}{d t} G_{\bar{L}, 0}(v(t))+\frac{b_{0}}{8} E_{\bar{L}}(v(t)) \leq 0 .
$$

Proof. Let $v \in X_{\delta, T}$ be a local solution to $(\mathrm{DW})_{\lambda}$. From (16), $C_{0}$ satisfies $C_{0} \geq d$. Thus using lemma 3.1 for $\mu=0$ and

$$
D_{\bar{L}, 0}(v(t))=E_{L_{0}}^{1 / 2}\left(\partial_{t}^{\mu} v(t)\right) E_{\bar{L}}\left(\partial_{t}^{\mu} v(t)\right) \leq \delta E_{\bar{L}}\left(\partial_{t}^{\mu} v(t)\right),
$$

we have

$$
\frac{d}{d t} G_{\bar{L}, 0}(v(t))+\frac{b_{0}}{2} E_{\bar{L}}(v(t)) \leq C\left(\lambda+\frac{\delta}{\lambda}\right) E_{\bar{L}}(v(t))+\frac{2\|B\|_{\infty} b_{0}^{2} R^{2}}{C_{0}} E_{\bar{L}}(v(t)) .
$$

From (16), we also obtain

$$
\frac{2\|B\|_{\infty} b_{0}^{2} R^{2}}{C_{0}} \leq \frac{b_{0}}{8}
$$


We choose sufficiently small constants $\lambda$ and $\delta$ such that

$$
C\left(\lambda+\frac{\delta}{\lambda}\right) \leq \frac{b_{0}}{4}
$$

then we have

$$
\frac{d}{d t} G_{\bar{L}, 0}(v(t))+\frac{b_{0}}{8} E_{\bar{L}}(v(t)) \leq 0 .
$$

This completes the proof of lemma 3.3.

Corollary 3.4. Let $L_{0} \leq \bar{L} \leq L$, and $\lambda$ and $\delta$ be sufficiently small constants in lemma 3.3. Then there exists a constant $C^{*}$ depending on $\lambda$ such that for any $T>0$ and a local solution $v \in X_{\delta, T}$ to $(\mathrm{DW})_{\lambda}$ satisfy

$$
\|v(t)\|_{2}^{2}+E_{\bar{L}}(v(t))+\int_{0}^{t} E_{\bar{L}}(v(s)) d s \leq C^{*}\left\{\|v(0)\|_{2}^{2}+E_{\bar{L}}(v(0))\right\}
$$

and

$$
\|v(t)\|_{H^{L_{0}}}^{2}+\left\|\partial_{t} v(t)\right\|_{H^{L_{0}-1}}^{2} \leq C^{*}\left\{\|v(0)\|_{H^{L_{0}}}^{2}+\left\|\partial_{t} v(0)\right\|_{H^{L_{0}-1}}^{2}\right\}
$$

Proof. The estimate (31) is verified by (30). Thus we should prove (30). Integrating $(29)$ over $[0, t]$ we, get

$$
G_{\bar{L}, 0}(v(t))+\frac{b_{0}}{8} \int_{0}^{t} E_{\bar{L}}(v(s)) d s \leq G_{\bar{L}, \mu}(v(0)) .
$$

Then using lemma 3.2, there exists a constant $C>0$ such that

$$
\frac{1}{C}\left\{\lambda\|v(t)\|_{2}^{2}+\frac{1}{\lambda} E_{l}(v(t))\right\}+\frac{b_{0}}{8} \int_{0}^{t} E_{l}(v(s)) d s \leq C\left\{\lambda\|v(0)\|_{2}^{2}+\frac{1}{\lambda} E_{\bar{L}}(v(0))\right\} .
$$

We rearrange coefficient and define $C^{*}$ depend on $\lambda$, it holds that (30).

\subsection{Global existence}

The following local existence theorem is known (see [10], [12], [16]).

Lemma 3.5. Let $L \geq L_{0}=[d / 2]+3, \lambda>0$ and $\left(v_{0}, v_{1}\right) \in H^{L} \times H^{L-1}$. For any sufficiently small constant $\varepsilon>0$ there exist constants $0<t_{0}$ and $0<\eta \leq 1$ such that if

$$
\left\|v_{0}\right\|_{H^{L_{0}}}^{2}+\left\|v_{1}\right\|_{H^{L_{0}-1}}^{2} \leq \eta \varepsilon^{2}
$$


then $(\mathrm{DW})_{\lambda}$ has a unique local solution $v \in \bigcap_{j=0}^{L} C^{j}\left(\left[0, t_{0}\right] ; H^{L-j}\right)$ and the $v$ satisfies

$$
E_{L_{0}}(v(t)) \leq \varepsilon^{2} \quad\left(0 \leq t \leq t_{0}\right) .
$$

Using lemma 3.5 and corollary 3.4 , we can prove a global existence theorem for $(\mathrm{DW})_{\lambda}$.

Theorem 3.6. Let $L \geq L_{0}=[d / 2]+3, \lambda$ and $\delta$ are sufficiently small constants. There exists a small constant $\delta^{*}>0$ such that if the initial data $\left(v_{0}, v_{1}\right) \in H^{L} \times H^{L-1}$ satisfy

$$
\left\|v_{0}\right\|_{H^{L_{0}}}^{2}+\left\|v_{1}\right\|_{H^{L_{0}-1}}^{2} \leq \delta^{*},
$$

then $(\mathrm{DW})_{\lambda}$ has a unique global solution $v \in X_{\delta}$.

Proof. Let $\lambda$ and $\delta>0$ are sufficiently small constants for which corollary 3.4 holds. Furthermore let $0<\varepsilon \leq \delta, 0<t_{0}$ and $0<\eta \leq 1$ be the constants given in lemma 3.5. Now we define

$$
\delta^{*}=\min \left\{\eta \varepsilon^{2}, \frac{\eta \varepsilon^{2}}{C^{*}}\right\}
$$

where the constant $C^{*}$ is given in corollary 3.4. We assume that $\left(v_{0}, v_{1}\right) \in$ $H^{L} \times H^{L-1}$ satisfy

$$
\left\|v_{0}\right\|_{H^{L_{0}}}^{2}+\left\|v_{1}\right\|_{H^{L_{0}-1}}^{2} \leq \delta^{*} .
$$

Lemma 3.5 yields that there exists $v \in \bigcap_{j=0}^{L} C^{j}\left(\left[0, t_{0}\right] ; H^{L-j}\right)$ such that $v$ is a unique local solution to $(\mathrm{DW})_{\lambda}$ and satisfies

$$
E_{L_{0}}(v(t)) \leq \varepsilon^{2} \leq \delta^{2} \quad\left(0 \leq t \leq t_{0}\right) .
$$

Because of $v \in X_{\delta, t_{0}}$ and corollary 3.4, it holds that

$$
\|v(t)\|_{H^{L_{0}}}^{2}+\left\|\partial_{t} v(t)\right\|_{H^{L_{0}-1}}^{2} \leq C^{*}\left\{\left\|v_{0}\right\|_{H^{L_{0}}}^{2}+\left\|v_{1}\right\|_{H^{L_{0}-1}}^{2}\right\} \leq \eta \varepsilon^{2} \quad\left(t \in\left[0, t_{0}\right]\right) .
$$

Thus we can use lemma 3.5 in $t=t_{0}$. The solution $v$ is uniquely extended to $\bigcap_{j=0}^{L} C^{j}\left(\left[0,2 t_{0}\right] ; H^{L-j}\right)$ and satisfies

$$
E_{L_{0}}(v(t)) \leq \varepsilon^{2} \leq \delta^{2} \quad\left(0 \leq t \leq 2 t_{0}\right) .
$$

Because of $v \in X_{\delta, 2 t_{0}}$ we can use corollary 3.4 again. Then $v$ satisfies

$$
\|v(t)\|_{H^{L_{0}}}^{2}+\left\|\partial_{t} v(t)\right\|_{H^{L_{0}-1}}^{2} \leq C^{*}\left\{\left\|v_{0}\right\|_{H^{L_{0}}}^{2}+\left\|v_{1}\right\|_{H^{L_{0}-1}}^{2}\right\} \leq \eta \varepsilon^{2} \quad\left(t \in\left[0,2 t_{0}\right]\right) .
$$

Thus we can use lemma 3.5 in $t=2 t_{0}$. 
Repeating this argument, we can uniquely extend $v$ to a global solution to $(\mathrm{DW})_{\lambda}$, furthermore the $v$ satisfies

$$
E_{L_{0}}(v(t)) \leq \delta^{2} \quad(t \in[0, \infty)) .
$$

This completes the proof of theorem 3.6.

Proof of theorem 1.1. Let $\lambda$ and $\delta^{*}$ are the small constants in theorem 3.6. We define $\hat{\delta}=\lambda^{d+1} \delta^{*}$ and assume the initial data $\left(u_{0}, u_{1}\right) \in H^{L} \times H^{L-1}$ satisfy

$$
\left\|u_{0}\right\|_{H^{L_{0}}}^{2}+\left\|u_{1}\right\|_{H^{L_{0}-1}}^{2} \leq \hat{\delta}
$$

Now we define

$$
v_{0}(x)=\frac{1}{\lambda} u_{0}(\lambda x), \quad v_{1}(x)=u_{1}(\lambda x)
$$

then $\left(v_{0}, v_{1}\right)$ satisfy

$$
\begin{aligned}
\left\|v_{0}\right\|_{H^{L_{0}}}^{2}+\left\|v_{1}\right\|_{H^{L_{0}-1}}^{2} & =\sum_{|a| \leq L_{0}} \lambda^{2(|a|-1)} \int_{\boldsymbol{R}^{d}}\left|\nabla^{a} u_{0}(\lambda x)\right|^{2} d x+\sum_{|a| \leq L_{0}-1} \lambda^{2|a|} \int_{\boldsymbol{R}^{d}}\left|\nabla^{a} u_{1}(\lambda x)\right|^{2} d x \\
& =\sum_{|a| \leq L_{0}} \lambda^{2(|a|-1)-d} \int_{\boldsymbol{R}^{d}}\left|\nabla^{a} u_{0}(x)\right|^{2} d x+\sum_{|a| \leq L_{0}-1} \lambda^{2|a|-d} \int_{\boldsymbol{R}^{d}}\left|\nabla^{a} u_{1}(x)\right|^{2} d x \\
& \leq \lambda^{-d-1}\left\{\sum_{|a| \leq L_{0}} \int_{\boldsymbol{R}^{d}}\left|\nabla^{a} u_{0}(x)\right|^{2} d x+\sum_{|a| \leq L_{0}-1} \int_{\boldsymbol{R}^{d}}\left|\nabla^{a} u_{1}(x)\right|^{2} d x\right\} \\
& =\lambda^{-d-1}\left\{\left\|u_{0}\right\|_{H^{L_{0}}}^{2}+\left\|u_{1}\right\|_{H^{L_{0}-1}}^{2}\right\} \leq \delta^{*} .
\end{aligned}
$$

From theorem 3.6, there exists a unique global solution $v$ to $(\mathrm{DW})_{\lambda}$ in $\bigcap_{j=0}^{L} C^{j}\left([0, \infty) ; H^{L-j}\right)$. We define

$$
u(t, x)=\lambda v\left(\frac{t}{\lambda}, \frac{x}{\lambda}\right),
$$

then $u \in \bigcap_{j=0}^{L} C^{j}\left([0, \infty) ; H^{L-j}\right)$ and the $u$ satisfies (DW).

As regard to uniqueness, if $u$ and $u^{\prime}$ are solutions to (DW) then rescaling functions $u_{\lambda}$ and $u_{\lambda}^{\prime}$ are solutions to $(\mathrm{DW})_{\lambda}$. From theorem 3.6 we got the uniqueness of $(\mathrm{DW})_{\lambda}$, so we obtain $u_{\lambda}=u_{\lambda}^{\prime}$, thus $u=u^{\prime}$.

\section{Decay estimates}

The goal of this section is to show theorem 4.1. We say that $f$ satisfies the property (H1)', (H2)' ${ }^{\prime}$ or (H3)' if and only if 
(H1)' $d \geq 3$ and there exists $1 \leq p \leq 2 d /(d+2)$ such that $f \in L^{p}$,

(H2)' $d \geq 3$ and $|\cdot| f \in L^{2}$,

(H3) $)^{\prime} d=1$ or $2,|\cdot| f \in L^{1}$ and

$$
\int_{\boldsymbol{R}^{d}} f(x) d x=0 .
$$

We prove the decay estimates for $(\mathrm{DW})_{\lambda}$ as follow:

Theorem 4.1. In addition to the assumptions in theorem 3.6, we assume that $B_{\lambda} v_{0}+v_{1}$ satisfies one of the $\left.\mathbf{( H 1}\right)^{\prime}-(\mathbf{H 3})^{\prime}$. Then for any $i\left(0 \leq i \leq L-L_{0}\right)$, there exists a constant $E_{0}$ depending on $\lambda, v_{0}$ and $v_{1}$ such that the global solution $v \in X_{\delta}$ to $(\mathrm{DW})_{\lambda}$ satisfies

$$
(1+t)^{2 i+1}\left\{\left\|\partial_{t}^{i} v(t)\right\|_{2}^{2}+E_{L-i}\left(\partial_{t}^{i} v(t)\right)\right\}+\int_{0}^{t}(1+s)^{2 i+1} E_{L-i}\left(\partial_{t}^{i} v(s)\right) d s \leq E_{0}
$$

and

$$
(1+t)^{2 i+2} E\left(\partial_{t}^{i} v(t)\right)+\int_{0}^{t}(1+s)^{2 i+2}\left\langle\partial_{t}^{i+1} v(s), B_{\lambda} \partial_{t}^{i+1} v(s)\right\rangle \leq E_{0} .
$$

Using theorem 4.1, we can prove theorem 1.2.

Proof of theorem 1.2. We assume that theorem 4.1 is true. From theorem 1.1 there exists a constant $\hat{\delta}$ such that if the initial data $\left(u_{0}, u_{1}\right)$ satisfies

$$
\left\|u_{0}\right\|_{H^{L_{0}}}^{2}+\left\|u_{1}\right\|_{H^{L_{0}-1}}^{2} \leq \hat{\delta}
$$

then (DW) has a unique global solution $u \in \bigcap_{j=0}^{L} C^{j}\left([0, \infty) ; H^{L-j}\right)$. Now we define $v=\lambda^{-1} u(\lambda t, \lambda x)$ then $v$ satisfies

$$
(\mathrm{DW})_{\lambda} \begin{cases}\left(\partial_{t}^{2}-\triangle+B_{\lambda}(x) \partial_{t}\right) v(t, x)=N[v, v](t, x), & (t, x) \in[0, \infty) \times \boldsymbol{R}^{d}, \\ v(0, x)=v_{0}(x), \partial_{t} v_{\lambda}(0, x)=v_{1}, & x \in \boldsymbol{R}^{d},\end{cases}
$$

where $v_{0}(x)=\lambda^{-1} u_{0}(\lambda x)$ and $v_{1}(x)=u_{1}(\lambda x)$. If $B u_{0}+u_{1}$ satisfies (H1), (H2) or (H3), then $B_{\lambda} v_{0}+v_{1}$ satisfies (H1)', (H2)' or (H3)'. Thus we can use theorem 4.1. For any $\mu\left(0 \leq \mu \leq L-L_{0}\right)$, there exists a constant $E_{0}$ depending on $\lambda, v_{0}$ and $v_{1}$ such that $v$ satisfies

$$
\left\|\partial_{t}^{\mu} v(\tau)\right\|_{2}^{2}+E_{L-\mu}\left(\partial_{t}^{\mu} v(\tau)\right) \leq E_{0}(1+\tau)^{-2 \mu-1}
$$

and

$$
E\left(\partial_{t}^{\mu} v(\tau)\right) \leq E_{0}(1+\tau)^{-2 \mu-2}
$$

Replacing $v(t, x)=\lambda^{-1} u(\lambda t, \lambda x)$ and $t=\lambda \tau$, we get (2) and (3). 
Using (2) and lemma 2.1, the estimate (4) is clear. Thus we prove (5). Let $L_{0}<L$. The global solution $u$ to (DW) satisfies

$$
\begin{aligned}
\|\Delta u(t)\|_{2} & =\left\|\partial_{t}^{2} u(t)+\partial_{t} u(t)-N[u, u](t)\right\|_{2} \\
& \leq\left\|\partial_{t}^{2} u(t)\right\|_{2}+\left\|\partial_{t} u(t)\right\|_{2}+\|N[u, u](t)\|_{2} .
\end{aligned}
$$

Because of $L_{0}<L$, we can use (2) and (3) to $\mu=1$. Then we obtain

$$
\begin{gathered}
\left\|\partial_{t} u\right\|_{2}^{2} \leq E_{0}(1+t)^{-3}, \\
\left\|\partial_{t}^{2} u\right\|_{2}^{2} \leq E_{0}(1+t)^{-4} .
\end{gathered}
$$

Furthermore using (2) and (3), we obtain

$$
\begin{aligned}
\|N[u, u](t)\|_{2}^{2} & \leq \sum_{i, j, k, l, m, n=1}^{d}\left|N_{l m n}^{i j k}\right|\left\|\partial_{l}\left(\partial_{m} u^{j} \partial_{n} u^{k}\right)\right\|_{2}^{2} \\
& \leq C\left\|\nabla^{2} u(t)\right\|_{\infty}^{2}\|\nabla u(t)\|_{2}^{2} \\
& \leq C E_{L_{0}}(u(t)) E(u(t)) \\
& \leq C E_{0}(1+t)^{-3} .
\end{aligned}
$$

Thus the estimate (5) holds from (38).

\subsection{Proof of theorem 4.1}

Let $v$ be the global solution to $(\mathrm{DW})_{\lambda}$ and define

$$
w(t, x)=\int_{0}^{t} v(s, x) d s .
$$

Then $w$ satisfies

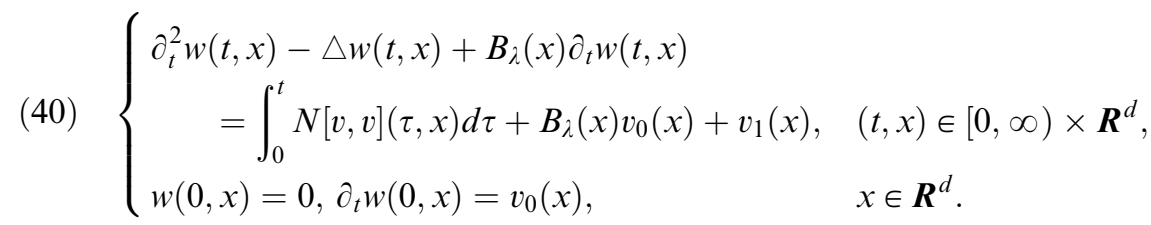

We remark $\partial_{t} w=v$ and $E_{L_{0}-1}(w(t))$ are well-defined in $[0, \infty)$ because of corollary 3.4.

We prove the energy estimate of $w$ under the assumption which $B_{\lambda} v_{0}+v_{1}$ satisfies one of the $(\mathbf{H 1})^{\prime}-(\mathbf{H 3})^{\prime}$. We prepare the next lemma. 
Lemma 4.2. Let $f \in H^{L_{0}-1}$ satisfies one of the $\left.(\mathbf{H 1})^{\prime}-\mathbf{( H 3}\right)^{\prime}$. Then for any $g \in H^{L_{0}-1}$, there exists a constant $E_{0}$ depending on $f$ such that

$$
\langle g, f\rangle \leq E_{0}\|\nabla g\|_{H^{L_{0}-2}} .
$$

Proof. First we assume that $f$ satisfies (H1)'. Then $f \in L^{2 d /(d+2)}$ because $f \in H^{L_{0}-1} \subset L^{\infty}$ and $f \in L^{p} \quad(1 \leq p \leq 2 d /(d+2))$. On the other hand, it holds from lemma 2.5 that

$$
\|g\|_{L^{2 d /(d-2)}} \leq C\|\nabla g\|_{2} .
$$

Thus using Hölder inequality, we get

$$
\langle g, f\rangle \leq\|f\|_{L^{2 d /(d+2)}}\|g\|_{L^{2 d /(d-2)}} \leq C\|f\|_{L^{2 d /(d+2)}}\|\nabla g\|_{2} \leq C\|f\|_{L^{2 d /(d+2)}}\|\nabla g\|_{H^{L_{0}-2}} .
$$

This means (41).

Next we assume that $f$ satisfies (H2)'. Then using lemma 2.4, we get

$$
\langle g, f\rangle \leq\||\cdot| f\|_{2}\left\|\frac{g}{|\cdot|}\right\|_{2} \leq C\||\cdot| f\|_{2}\|\nabla g\|_{2} \leq C\||\cdot| f\|_{2}\|\nabla g\|_{H^{L_{0}-2}} .
$$

Thus we have (41).

Finally we assume that $f$ satisfies (H3)'. Because of $g \in H^{L_{0}-1} \subset C^{1}\left(\boldsymbol{R}^{d}\right)$, we have

$$
|g(x)-g(0)|=\left|\int_{0}^{1} \frac{d}{d \theta} g(\theta x) d \theta\right|=\left|\int_{0}^{1} x \cdot \nabla g(\theta x) d \theta\right| \leq|x|\|\nabla g\|_{\infty} \quad \text { a.e. } x .
$$

Then using $\int_{\boldsymbol{R}^{d}} f(x) d x=0$, we obtain

$$
\begin{aligned}
|\langle g, f\rangle| & =\left|\int_{\boldsymbol{R}^{d}}(g(x)-g(0)) f(x) d x\right| \leq \int_{\boldsymbol{R}^{d}} \mid(g(x)-g(0)|| f(x) \mid d x \\
& \leq\|\nabla g\|_{\infty} \int_{\boldsymbol{R}^{d}}|x||f(x)| d x \leq C\|\nabla g\|_{H^{L_{0}-2}}\||\cdot| f\|_{1} .
\end{aligned}
$$

Thus we get (41).

We need the estimate $\int_{0}^{\infty}\|v(t)\|_{2}^{2} d t<\infty$. In order to prove this property, we use the idea in Ikehata [5].

Proposition 4.3. In addition to the assumptions theorem 3.6, we assume $B_{\lambda} v_{0}+v_{1}$ satisfies one of the $(\mathbf{H 1})^{\prime}-(\mathbf{H 3})^{\prime}$. Then there exists a constant $E_{0}$ depending on $\lambda, v_{0}$ and $v_{1}$ such that the global solution $v \in X_{\delta}$ to $(\mathrm{DW})_{\lambda}$ satisfies

$$
\int_{0}^{t}\|v(s)\|_{2}^{2} d s \leq E_{0}, \quad(t \in[0, \infty)) .
$$

Proof. Let $v$ be the global solution to $(\mathrm{DW})_{\lambda}$ and $w$ be defined by (39). Using (40), we have 


$$
\begin{aligned}
\frac{d}{d t} E_{L_{0}-1}(w(t))= & \sum_{|a| \leq L_{0}-2}\left\langle\partial_{t} \nabla^{a} w(t), \partial_{t}^{2} \nabla^{a} w(t)\right\rangle-\sum_{|a| \leq L_{0}-2}\left\langle\partial_{t} \nabla^{a} w(t), \Delta \nabla^{a} w(t)\right\rangle \\
= & -\sum_{|a| \leq L_{0}-2}\left\langle\partial_{t} \nabla^{a} w(t), \nabla^{a}\left(B_{\lambda} \partial_{t} w(t)\right)\right\rangle \\
& +\sum_{|a| \leq L_{0}-2}\left\langle\partial_{t} \nabla^{a} w(t), \int_{0}^{t} \nabla^{a} N[v, v] d \tau\right\rangle \\
& +\sum_{|a| \leq L_{0}-2}\left\langle\partial_{t} \nabla^{a} w(t), \nabla^{a}\left(B_{\lambda} v_{0}+v_{1}\right)\right\rangle \\
= & \left.-\sum_{|a| \leq L_{0}-2}\left\langle\partial_{t} \nabla^{a} w(t), B_{\lambda} \partial_{t} \nabla^{a} w(t)\right)\right\rangle \\
& \left.-\sum_{1 \leq|a| \leq L_{0}-2} \sum_{b \leq a}^{b \neq 0}\left(\begin{array}{l}
a \\
b \neq 0
\end{array}\right)\left\langle\partial_{t} \nabla^{a} w(t), \nabla^{b} B_{\lambda} \partial_{t} \nabla^{a-b} w(t)\right)\right\rangle \\
& +\sum_{|a| \leq L_{0}-2} \frac{d}{d t}\left\langle\nabla^{a} w(t), \int_{0}^{t} \nabla^{a} N[v, v] d \tau\right\rangle \\
& -\sum_{|a| \leq L_{0}-2}\left\langle\nabla^{a} w(t), \nabla^{a} N[v, v](t)\right\rangle \\
& +\sum_{|a| \leq L_{0}-2} \frac{d}{d t}\left\langle\nabla^{a} w(t), \nabla^{a}\left(B_{\lambda} v_{0}+v_{1}\right)\right\rangle .
\end{aligned}
$$

Then integrating it over $[0, t]$, we get

$$
\begin{aligned}
E_{L_{0}-1}(w(t)) & +\sum_{|a| \leq L_{0}-2} \int_{0}^{t}\left\langle\nabla^{a} v(s), B_{\lambda} \nabla^{a} v(s)\right\rangle d s \\
\leq & \left.\frac{1}{2}\left\|v_{0}\right\|_{H^{L_{0}-2}}^{2}-\sum_{1 \leq|a| \leq L_{0}-2} \sum_{\substack{b \leq a \\
b \neq 0}}\left(\begin{array}{l}
a \\
b
\end{array}\right) \int_{0}^{t}\left\langle\nabla^{a} v(s), \nabla^{b} B_{\lambda} \nabla^{a-b} v(s)\right)\right\rangle d s \\
& +\sum_{|a| \leq L_{0}-2}\left\langle\nabla^{a} w(t), \int_{0}^{t} \nabla^{a} N[v, v](s) d s\right\rangle \\
& -\sum_{|a| \leq L_{0}-2} \int_{0}^{t}\left\langle\nabla^{a} w(s), \nabla^{a} N[v, v](s)\right\rangle d s \\
& +\sum_{|a| \leq L_{0}-2}\left\langle\nabla^{a} w(t), \nabla^{a}\left(B_{\lambda} v_{0}+v_{1}\right)\right\rangle \\
= & \frac{1}{2}\left\|v_{0}\right\|_{H^{L_{0}-2}}^{2}+A_{1}+A_{2}+A_{3}+A_{4} .
\end{aligned}
$$


We estimate from $A_{1}$ to $A_{4}$. Using lemma 2.3 , (B3) $)_{\lambda}$ and smallness of $\lambda$, we get

$$
\begin{aligned}
\left|A_{1}\right| & \leq \sum_{1 \leq|a| \leq L_{0}-2} \sum_{\substack{b \leq a \\
b \neq 0}}\left(\begin{array}{l}
a \\
b
\end{array}\right) \int_{0}^{t}\left\|\nabla^{a} v(s)\right\|_{2}\left\|\nabla^{b} B_{\lambda}\right\|_{\infty}\left\|\nabla^{a-b} v(s)\right\|_{2} d s \\
& \leq \lambda^{2} C \sum_{1 \leq|a| \leq L_{0}-2} \sum_{\substack{b \leq a \\
b \neq 0}} \int_{0}^{t}\left\|\nabla^{a} v(s)\right\|_{2}\left\|\nabla^{a-b} v(s)\right\|_{2} d s \\
& \leq C \sum_{1 \leq|a| \leq L_{0}-2}\left(\int_{0}^{t}\left\|\nabla^{a} v(s)\right\|_{2}^{2} d s+\lambda^{4} C \sum_{\substack{b \leq a \\
b \neq 0}} \int_{0}^{t}\left\|\nabla^{a-b} v(s)\right\|_{2}^{2} d s\right) \\
& \leq C \int_{0}^{t} E_{L_{0}-2}(v(s)) d s+\lambda^{2} C \sum_{\substack{1 \leq|a| \leq L_{0}-2 \\
b \leq a \\
b \neq 0}}\left\{\int_{0}^{t}\left\langle\nabla^{a-b} v(s), B_{\lambda} \nabla^{a-b} v(s)\right\rangle d s\right. \\
& \leq C \int_{0}^{t} E_{L_{0}}(v(s)) d s+\frac{1}{4} \sum_{|a| \leq L_{0}-2} \int_{0}^{t}\left\langle\nabla^{a} v(s), B_{\lambda} \nabla^{a} v(s)\right\rangle d s .
\end{aligned}
$$

Next we define

$$
M(t)=\sup _{0 \leq s \leq t} E_{L_{0}-1}(w(s)) .
$$

Using $M(t)$ and lemma 2.2, we have

$$
\begin{aligned}
\left|A_{2}\right| & =\sum_{|a| \leq L_{0}-2}\left|\left\langle\nabla^{a} w(t), \int_{0}^{t} \nabla^{a} N[v, v](s) d s\right\rangle\right| \\
& =\sum_{|a| \leq L_{0}-2}\left|\sum_{i, j, k, l, m, n=1}^{d} N_{l m n}^{i j k} \int_{0}^{t} \int_{\boldsymbol{R}^{d}} \nabla^{a} w^{i}(t) \partial_{l} \nabla^{a}\left(\partial_{m} v^{j}(s) \partial_{n} v^{k}(s)\right) d x d s\right| \\
& =\sum_{|a| \leq L_{0}-2}\left|\sum_{i, j, k, l, m, n=1}^{d} N_{l m n}^{i j k} \int_{0}^{t} \int_{\boldsymbol{R}^{d}} \partial_{l} \nabla^{a} w^{i}(t) \nabla^{a}\left(\partial_{m} v^{j}(s) \partial_{n} v^{k}(s)\right) d x d s\right| \\
& \leq C \sum_{|a| \leq L_{0}-2} \int_{0}^{t}\left\|\nabla \nabla^{a} w(t)\right\|_{2} \sum_{j, k, m, n=1}^{d}\left\|\nabla^{a}\left(\partial_{m} v^{j}(s) \partial_{n} v^{k}(s)\right)\right\|_{2} d s \\
& \leq C(M(t))^{1 / 2} \int_{0}^{t} E_{L_{0}}(v(s)) d s
\end{aligned}
$$


and

$$
\begin{aligned}
\left|A_{3}\right| & =\sum_{|a| \leq L_{0}-2}\left|\int_{0}^{t}\left\langle\nabla^{a} w(s), \nabla^{a} N[v, v](s) d s\right\rangle\right| \\
& \leq \sum_{|a| \leq L_{0}-2}\left|\sum_{i, j, k, l, m, n=1}^{d} N_{l m n}^{i j k} \int_{0}^{t} \int_{\boldsymbol{R}^{d}} \nabla^{a} w^{i}(s) \partial_{l} \nabla^{a}\left(\partial_{m} v^{j}(s) \partial_{n} v^{k}(s)\right) d x d s\right| \\
& =\sum_{|a| \leq L_{0}-2}\left|\sum_{i, j, k, l, m, n=1}^{d} N_{l m n}^{i j k} \int_{0}^{t} \int_{\boldsymbol{R}^{d}} \partial_{l} \nabla^{a} w^{i}(s) \nabla^{a}\left(\partial_{m} v^{j}(s) \partial_{n} v^{k}(s)\right) d x d s\right| \\
& \leq C \sum_{|a| \leq L_{0}-2} \int_{0}^{t}\left\|\nabla \nabla^{a} w(s)\right\|_{2} \sum_{j, k, m, n=1}^{d}\left\|\nabla^{a}\left(\partial_{m} v^{j}(s) \partial_{n} v^{k}(s)\right)\right\|_{2} d s \\
& \leq C(M(t))^{1 / 2} \int_{0}^{t} E_{L_{0}}(v(s)) d s .
\end{aligned}
$$

Using lemma 4.2, we get

$$
\begin{aligned}
A_{4} & =\left\langle w(t), B_{\lambda} v_{0}+v_{1}\right\rangle+\sum_{1 \leq|a| \leq L_{0}-2}\left\langle\nabla^{a} w(t), \nabla^{a}\left(B_{\lambda} v_{0}+v_{1}\right)\right\rangle \\
& \leq E_{0}\|\nabla w(t)\|_{H^{L_{0}-2}}+\sum_{1 \leq|a| \leq L_{0}-2}\left\|\nabla^{a} w(t)\right\|_{2}\left\|\nabla^{a}\left(B_{\lambda} v_{0}+v_{1}\right)\right\|_{2} \\
& \leq E_{0} E_{L_{0}-1}^{1 / 2}(w(t)) \leq E_{0}^{2}+\frac{1}{4} E_{L_{0}-1}(w(t))
\end{aligned}
$$

where $E_{0}$ depend on $\lambda, v_{0}$ and $v_{1}$. Corollary 3.4 implies that there exists a constant $C^{*}>0$ such that

$$
\int_{0}^{t} E_{L_{0}}(v(s)) d s \leq C^{*}\left\{\|v(0)\|_{2}^{2}+E_{L_{0}}(v(0))\right\} .
$$

From (43) and the estimates of $A_{1}-A_{4}$, it holds that

$$
E_{L_{0}-1}(w(t))+\sum_{|a| \leq L_{0}-2} \int_{0}^{t}\left\langle\nabla^{a} v(s), B_{\lambda} \nabla^{a} v(s)\right\rangle d s \leq E_{0}+E_{0}(M(t))^{1 / 2} .
$$

From (45) we have

$$
M(t) \leq E_{0}+E_{0}(M(t))^{1 / 2} \quad(t \in[0, \infty)) .
$$

It means that $M(t)$ is bounded in $[0, \infty)$. Thus it holds from (45) that

$$
\int_{0}^{t}\left\langle v(s), B_{\lambda} v(s)\right\rangle d s \leq E_{0}
$$


Finally using (46), lemma 2.3 and Corollary 3.4, we have

$$
\int_{0}^{t}\|v(t)\|_{2}^{2} d s \leq \frac{C}{\lambda} \int_{0}^{t}\left\langle v(s), B_{\lambda} v(s)\right\rangle d s+\frac{C}{\lambda^{2}} \int_{0}^{t}\|\nabla v(s)\|_{2}^{2} d s \leq \frac{E_{0}}{\lambda}+\frac{E_{0}}{\lambda^{2}} .
$$

Thus we get (42).

Let prove theorem 4.1 by the induction. First we prove for $i=0$.

Theorem 4.4. In addition to the assumption theorem 3.6, we assume that $B_{\lambda} v_{0}+v_{1}$ satisfies one of the $(\mathbf{H 1})^{\prime}-(\mathbf{H 3})^{\prime}$. Then there exists constant $E_{0}$ depending on $\lambda, v_{0}$ and $v_{1}$ such that the global solution $v \in X_{\delta}$ to $(\mathrm{DW})_{\lambda}$ satisfies

$$
(1+t)\left\{\|v(t)\|_{2}^{2}+E_{L}(v(t))\right\}+\int_{0}^{t}(1+s) E_{L}(v(s)) d s \leq E_{0}
$$

and

$$
(1+t)^{2} E(v(t))+\int_{0}^{t}(1+s)^{2}\left\langle\partial_{t} v(s), B_{\lambda} \partial_{t} v(s)\right\rangle \leq E_{0} .
$$

Proof. First we prove (47). Using lemma 3.3 for $\bar{L}=L$, we obtain

$$
\begin{aligned}
\frac{d}{d t}\left\{(1+t) G_{L, 0}(v(t))\right\} & =G_{L, 0}(v(t))+(1+t) \frac{d}{d t} G_{L, 0}(v(t)) \\
& \leq G_{L, 0}(v(t))-\frac{b_{0}(1+t)}{8} E_{L}(v(t)) .
\end{aligned}
$$

Integrating it over $[0, t]$ and using lemma 3.2 , we get

$$
\begin{aligned}
& \frac{(1+t)}{C}\left\{\lambda\|v(t)\|_{2}^{2}+\frac{1}{\lambda} E_{L}(v(t))\right\}+\frac{b_{0}}{8} \int_{0}^{t}(1+s) E_{L}(v(s)) d s \\
& \quad \leq C\left\{\lambda\|v(0)\|_{2}^{2}+\frac{1}{\lambda} E_{L}(v(0))+\lambda \int_{0}^{t}\|v(s)\|_{2}^{2} d s+\frac{1}{\lambda} \int_{0}^{t} E_{L}(v(s)) d s\right\} .
\end{aligned}
$$

Then there exists a constant $E_{0}$ which depends on $\lambda, v_{0}$ and $v_{1}$ such that

$$
\begin{gathered}
(1+t)\left\{\|v(t)\|_{2}^{2}+E_{L}(v(t))\right\}+\int_{0}^{t}(1+s) E_{L}(v(s)) d s \\
\leq E_{0}+E_{0} \int_{0}^{t}\|v(s)\|_{2}^{2} d s+E_{0} \int_{0}^{t} E_{L}(v(s)) d s .
\end{gathered}
$$

Using proposition 4.3 and corollary 3.4 , we get 


$$
\begin{gathered}
(1+t)\left\{\lambda\|v(t)\|_{2}^{2}+E_{L}(v(t))\right\}+\int_{0}^{t}(1+s) E_{L}(v(s)) d s \\
\leq E_{0}+E_{0}^{2}+E_{0} C^{*}\left\{\|v(0)\|_{2}^{2}+E_{L}(v(0))\right\} .
\end{gathered}
$$

Rearranging $E_{0}$ if we need, we get (47).

Next we prove (48). It holds that

$$
\begin{aligned}
& \frac{d}{d t}\left\{(1+t)^{2} E(v(t))\right\} \\
&=2(1+t) E(v(t))+(1+t)^{2} \frac{d}{d t} E(v(t)) \\
&=2(1+t) E(v(t))+(1+t)^{2}\left\{\left\langle\partial_{t} v(t), \partial_{t}^{2} v(t)\right\rangle-\left\langle\partial_{t} v(t), \triangle v(t)\right\rangle\right\} \\
&=2(1+t) E(v(t))-(1+t)^{2}\left\langle\partial_{t} v(t), B_{\lambda} \partial_{t} v(t)\right\rangle \\
&+(1+t)^{2}\left\langle\partial_{t} v(t), N[v, v](t)\right\rangle .
\end{aligned}
$$

Using (50) and

$$
\left\langle\partial_{t} v(t), N[v, v](t)\right\rangle \leq C E^{1 / 2}(v(t)) E_{L}(v(t)),
$$

we get

$$
\begin{aligned}
& \frac{d}{d t}\left\{(1+t)^{2} E(v(t))\right\}+(1+t)^{2}\left\langle\partial_{t} v(t), B_{\lambda} \partial_{t} v(t)\right\rangle \\
& \quad \leq 2(1+t) E(v(t))+C(1+t)^{2} E^{1 / 2}(v(t)) E_{L}(v(t))
\end{aligned}
$$

Now we define

$$
M_{0}(t)=\sup _{0 \leq s \leq t}(1+s)^{2} E(v(s))
$$

Integrating (51) over $[0, \infty)$ and using (47), we obtain

$$
\begin{aligned}
(1+ & t)^{2} E(v(t))+\int_{0}^{t}(1+s)^{2}\left\langle\partial_{t} v(s), B_{\lambda} \partial_{t} v(s)\right\rangle d s \\
& \leq E(v(0))+2 \int_{0}^{t}(1+s) E(v(s)) d s+C \int_{0}^{t}(1+s)^{2} E^{1 / 2}(v(s)) E_{L}(v(s)) d s \\
& \leq E(v(0))+2 \int_{0}^{t}(1+s) E(v(s)) d s+C\left(M_{0}(t)\right)^{1 / 2} \int_{0}^{t}(1+s) E_{L}(v(s)) d s \\
& \leq E_{0}+E_{0}\left(M_{0}(t)\right)^{1 / 2}
\end{aligned}
$$


From (53) we have

$$
M_{0}(t) \leq E_{0}+E_{0}\left(M_{0}(t)\right)^{1 / 2},
$$

which means that $M_{0}(t)$ is bounded in $[0, \infty)$. Therefore it holds from (53) that

$$
(1+t)^{2} E(v(t))+\int_{0}^{t}(1+s)^{2}\left\langle\partial_{t} v(s), B_{\lambda} \partial_{t} v(s)\right\rangle \leq E_{0} .
$$

Thus we get (48).

Next assuming the decay estimate of $\partial_{t}^{i} v$ for $0 \leq i \leq \mu-1$, we show the decay estimate of $\partial_{t}^{\mu} v$. For the purpose, we need the following lemma:

Lemma 4.5. In addition to the assumption theorem 3.6, we assume that $B_{\lambda} v_{0}+v_{1}$ satisfies one of the (H1)'-(H3)'. Let $1 \leq \mu \leq L-L_{0}$ and assume that for any $0 \leq i \leq \mu-1$ estimates (36) and (37) in theorem 4.1 hold. Then there exists a constant $E_{0}$ depending on $\lambda, v_{0}$ and $v_{1}$ such that the global solution $v \in X_{\delta}$ to $(\mathrm{DW})_{\lambda}$ satisfies

$$
\frac{d}{d t} G_{L, \mu}(v(t))+\frac{b_{0}}{8} E_{L-\mu}\left(\partial_{t}^{\mu} v(t)\right) \leq E_{0} \sum_{v=1}^{\mu} E_{L-v}\left(\partial_{t}^{v} v(t)\right)(1+t)^{-2(\mu-v)-1}
$$

if $\lambda$ and $\delta$ in theorem 3.6 are chosen small enough.

Proof. From (18) in lemma 3.1 and the assumption of induction, it follows that

$$
\begin{aligned}
D_{L, \mu}(v(t)) & =E_{L-\mu}^{1 / 2}\left(\partial_{t}^{\mu} v(t)\right) \cdot\left(\sum_{v=0}^{\mu} E_{L_{0}}^{1 / 2}\left(\partial_{t}^{\mu-v} v(t)\right) E_{L-v}^{1 / 2}\left(\partial_{t}^{v} v(t)\right)\right) \\
& \leq \delta E_{L-\mu}\left(\partial_{t}^{\mu} v(t)\right)+E_{L-\mu}^{1 / 2}\left(\partial_{t}^{\mu} v(t)\right) \cdot\left(\sum_{v=0}^{\mu-1} E_{L_{0}}^{1 / 2}\left(\partial_{t}^{\mu-v} v(t)\right) E_{L-v}^{1 / 2}\left(\partial_{t}^{v} v(t)\right)\right) \\
& \leq 2 \delta E_{L-\mu}\left(\partial_{t}^{\mu} v(t)\right)+\frac{1}{4 \delta}\left(\sum_{v=0}^{\mu-1} E_{L_{0}}^{1 / 2}\left(\partial_{t}^{\mu-v} v(t)\right) E_{L-v}^{1 / 2}\left(\partial_{t}^{v} v(t)\right)\right)^{2} \\
& \leq 2 \delta E_{L-\mu}\left(\partial_{t}^{\mu} v(t)\right)+\frac{\mu}{4 \delta} \sum_{v=0}^{\mu-1} E_{L_{0}}\left(\partial_{t}^{\mu-v} v(t)\right) E_{L-v}\left(\partial_{t}^{v} v(t)\right) \\
& \leq 2 \delta E_{L-\mu}\left(\partial_{t}^{\mu} v(t)\right)+\frac{\mu E_{0}}{4 \delta} \sum_{v=0}^{\mu-1} E_{L-(\mu-v)}\left(\partial_{t}^{\mu-v} v(t)\right)(1+t)^{-2 v-1} \\
& =2 \delta E_{L-\mu}\left(\partial_{t}^{\mu} v(t)\right)+\frac{\mu E_{0}}{4 \delta} \sum_{v=1}^{\mu} E_{L-v}\left(\partial_{t}^{v} v(t)\right)(1+t)^{-2(\mu-v)-1} .
\end{aligned}
$$


The above estimate and lemma 3.1 imply that

$$
\begin{aligned}
\frac{d}{d t} G_{L, \mu}(v(t))+\frac{b_{0}}{2} E_{L-\mu}\left(\partial_{t}^{\mu} v(t)\right) \\
\leq \lambda C E_{L-\mu}\left(\partial_{t}^{\mu} v(t)\right)+\frac{C}{\lambda} D_{L, \mu}(v(t))+\frac{2\|B\|_{\infty} b_{0}^{2} R^{2}}{C_{0}} E_{L-\mu}\left(\partial_{t}^{\mu} v(t)\right) \\
\leq\left(\lambda C+\frac{2 \delta C}{\lambda}+\frac{2\|B\|_{\infty} b_{0}^{2} R^{2}}{C_{0}}\right) E_{L-\mu}\left(\partial_{t}^{\mu} v(t)\right) \\
\quad+\frac{C E_{0}}{\lambda \delta} \sum_{v=1}^{\mu} E_{L-v}\left(\partial_{t}^{v} v(t)\right)(1+t)^{-2(\mu-v)-1} .
\end{aligned}
$$

From (16), it follows that

$$
\frac{2\|B\|_{\infty} b_{0}^{2} R^{2}}{C_{0}} \leq \frac{b_{0}}{8}
$$

Choosing $\lambda$ and $\delta>0$ in theorem 3.6 sufficiently small enough if necessary, we obtain

$$
\lambda C+\frac{2 \delta C}{\lambda} \leq \frac{b_{0}}{4}
$$

From these estimates and (55), it holds that there exists a constant $E_{0}$ such that

$$
\frac{d}{d t} G_{L, \mu}(v(t))+\frac{b_{0}}{8} E_{L-\mu}\left(\partial_{t}^{\mu} v(t)\right) \leq E_{0} \sum_{v=1}^{\mu} E_{L-v}\left(\partial_{t}^{v} v(t)\right)(1+t)^{-2(\mu-v)-1} .
$$

This means (54). Hence we obtain lemma 4.5.

We complete to prove theorem 4.1. When $i=0$ we already proved (theorem 4.4), thus we assume $1 \leq \mu \leq L-L_{0}$ and for any $0 \leq i \leq \mu-1$ satisfy theorem 4.1. The goal is to show (36) and (37) to $i=\mu$.

First we prove (36) for $i=\mu$. Lemma 4.5 yields that

$$
\begin{aligned}
\frac{d}{d t}\left\{(1+t)^{2 \mu+1} G_{L, \mu}(v(t))\right\} \\
=(2 \mu+1)(1+t)^{2 \mu} G_{L, \mu}(v(t))+(1+t)^{2 \mu+1} \frac{d}{d t} G_{L, \mu}(v(t)) \\
\leq(2 \mu+1)(1+t)^{2 \mu} G_{L, \mu}(v(t))-(1+t)^{2 \mu+1} \frac{b_{0}}{8} E_{L-\mu}\left(\partial_{t}^{\mu} v(t)\right) \\
\quad+E_{0} \sum_{v=1}^{\mu} E_{L-v}\left(\partial_{t}^{v} v(t)\right)(1+t)^{2 v} .
\end{aligned}
$$


Integrating (56) over $[0, t]$, we get

$$
\begin{aligned}
(1+t)^{2 \mu+1} G_{L, \mu}(v(t))+\frac{b_{0}}{8} \int_{0}^{t}(1+s)^{2 \mu+1} E_{L-\mu}\left(\partial_{t}^{\mu} v(s)\right) d s \\
\leq G_{L, \mu}(v(0))+(2 \mu+1) \int_{0}^{t}(1+s)^{2 \mu} G_{L, \mu}(v(s)) d s \\
\quad+E_{0} \sum_{v=1}^{\mu} \int_{0}^{t}(1+s)^{2 v} E_{L-v}\left(\partial_{t}^{v} v(s)\right) d s .
\end{aligned}
$$

From (20) and (56), there exists a constant $E_{0}$ depending on $\lambda, v_{0}$ and $v_{1}$ such that

$$
\begin{aligned}
(1+t)^{2 \mu+1}\left\{\left\|\partial_{t}^{\mu} v(t)\right\|_{2}^{2}+E_{L-\mu}\left(\partial_{t}^{\mu} v(t)\right)\right\}+\int_{0}^{t}(1+s)^{2 \mu+1} E_{L-\mu}\left(\partial_{t}^{\mu} v(s)\right) d s \\
\leq E_{0}+E_{0} \int_{0}^{t}(1+s)^{2 \mu}\left\|\partial_{t}^{\mu} v(s)\right\|_{2}^{2} d s+E_{0} \int_{0}^{t}(1+t)^{2 \mu} E_{L-\mu}\left(\partial_{t}^{\mu} v(s)\right) d s \\
\quad+E_{0} \sum_{v=1}^{\mu-1} \int_{0}^{t}(1+s)^{2 v} E_{L-v}\left(\partial_{t}^{v} v(s)\right) d s .
\end{aligned}
$$

We estimate the right-side of (58). Using lemma 2.3 and (37) for $i=\mu-1$, we get

$$
\text { (59) } \begin{aligned}
\int_{0}^{t}(1 & +s)^{2 \mu}\left\|\partial_{t}^{\mu} v(s)\right\|_{2}^{2} d s \\
& \leq \frac{C_{1}}{\lambda} \int_{0}^{t}(1+s)^{2 \mu}\left\langle\partial_{t}^{\mu} v(s), B_{\lambda} \partial_{t}^{\mu} v(s)\right\rangle d s+\frac{C_{1}}{\lambda^{2}} \int_{0}^{t}(1+s)^{2 \mu}\left\|\nabla \partial_{t}^{\mu} v(s)\right\|_{2}^{2} d s \\
& \leq \frac{C_{1} E_{0}}{\lambda}+\frac{2 C_{1}}{\lambda^{2}} \int_{0}^{t}(1+s)^{2 \mu} E_{L-\mu}\left(\partial_{t}^{\mu} v(s)\right) d s .
\end{aligned}
$$

From the assumption of induction, it follows that (36) for $i=v$ with $v \leq \mu-1$ hold, which yields that

$$
\sum_{v=1}^{\mu-1} \int_{0}^{t}(1+s)^{2 v} E_{L-v}\left(\partial_{t}^{v} v(s)\right) d s \leq(\mu-1) E_{0} .
$$

Using (58), (59) and (60), we obtain

$$
\begin{gathered}
(61)(1+t)^{2 \mu+1}\left\{\left\|\partial_{t}^{\mu} v(t)\right\|_{2}^{2}+E_{L-\mu}\left(\partial_{t}^{\mu} v(t)\right)\right\}+\int_{0}^{t}(1+s)^{2 \mu+1} E_{L-\mu}\left(\partial_{t}^{\mu} v(s)\right) d s \\
\leq E_{0}+E_{0} \int_{0}^{t}(1+t)^{2 \mu} E_{L-\mu}\left(\partial_{t}^{\mu} v(s)\right) d s .
\end{gathered}
$$


We choose a constant $t^{*}$ such that $2 E_{0}\left(1+t^{*}\right)^{-1} \leq 1$ then we get

(62) $\quad E_{0} \int_{0}^{t}(1+s)^{2 \mu} E_{L-\mu}\left(\partial_{t}^{\mu} v(s)\right) d s$

$$
\begin{aligned}
= & E_{0} \int_{0}^{t^{*}}(1+s)^{2 \mu} E_{L-\mu}\left(\partial_{t}^{\mu} v(s)\right) d s \\
& +E_{0} \int_{t^{*}}^{t}(1+s)^{2 \mu+1}(1+s)^{-1} E_{L-\mu}\left(\partial_{t}^{\mu} v(s)\right) d s \\
\leq & \frac{E_{0}\left(1+t^{*}\right)^{2 \mu+1}}{2 \mu+1} \sup _{0 \leq s \leq t^{*}} E_{L-\mu}\left(\partial_{t}^{\mu} v(s)\right)+\frac{1}{2} \int_{0}^{t}(1+t)^{2 \mu+1} E_{L-\mu}\left(\partial_{t}^{\mu} v(s)\right) d s .
\end{aligned}
$$

Now

$$
\frac{E_{0}\left(1+t^{*}\right)^{2 \mu+1}}{2 \mu+1} \sup _{0 \leq s \leq t^{*}} E_{L-\mu}\left(\partial_{t}^{\mu} v(s)\right)
$$

can include to $E_{0}$ because the constant is depended on $\lambda, u_{0}$ and $u_{1}$. Therefore using (61) and (62), we get (36) to $i=\mu$.

Next we prove (37) to $i=\mu$. For the solution $v$ to $(\mathrm{DW})_{\lambda}$ holds that

$$
\begin{aligned}
& \frac{d}{d t}\left\{(1+t)^{2 \mu+2} E\left(\partial_{t}^{\mu} v(t)\right)\right\} \\
&=(2 \mu+2)(1+t)^{2 \mu+1} E\left(\partial_{t}^{\mu} v(t)\right)+(1+t)^{2 \mu+2} \frac{d}{d t} E\left(\partial_{t}^{\mu} v(t)\right) \\
& \leq(2 \mu+2)(1+t)^{2 \mu+1} E_{L-\mu}\left(\partial_{t}^{\mu} v(t)\right)-(1+t)^{2 \mu+2}\left\langle\partial_{t}^{\mu+1} v(t), B_{\lambda} \partial_{t}^{\mu+1} v(t)\right\rangle \\
&+(1+t)^{2 \mu+2}\left\langle\partial_{t}^{\mu+1} v(t), \partial_{t}^{\mu} N[v, v](t)\right\rangle .
\end{aligned}
$$

Using the above estimate and

$$
\begin{aligned}
\left\langle\partial_{t}^{\mu+1} v(t), \partial_{t}^{\mu} N[v, v](t)\right\rangle & \leq C\left\|\partial_{t}^{\mu+1} v(t)\right\|_{2} \sum_{v=0}^{\mu}\left(\begin{array}{c}
\mu \\
v
\end{array}\right)\left\|\partial_{t}^{v} \nabla^{2} v(t)\right\|_{\infty}\left\|\partial_{t}^{\mu-v} \nabla v(t)\right\|_{2} \\
& \leq C E^{1 / 2}\left(\partial_{t}^{\mu} v(t)\right) \sum_{v=0}^{\mu} E_{L-v}^{1 / 2}\left(\partial_{t}^{v} v(t)\right) E_{L-(\mu-v)}^{1 / 2}\left(\partial_{t}^{\mu-v} v(t)\right)
\end{aligned}
$$

we obtain

$$
\begin{aligned}
& \frac{d}{d t}\left\{(1+t)^{2 \mu+2} E\left(\partial_{t}^{\mu} v(t)\right)\right\}+(1+t)^{2 \mu+2}\left\langle\partial_{t}^{\mu+1} v(t), B_{\lambda} \partial_{t}^{\mu+1} v(t)\right\rangle \\
& \leq C(1+t)^{2 \mu+1} E_{L-\mu}\left(\partial_{t}^{\mu} v(t)\right) \\
&+C(1+t)^{2 \mu+2} E^{1 / 2}\left(\partial_{t}^{\mu} v(t)\right) \sum_{v=0}^{\mu} E_{L-v}^{1 / 2}\left(\partial_{t}^{v} v(t)\right) E_{L-(\mu-v)}^{1 / 2}\left(\partial_{t}^{\mu-v} v(t)\right)
\end{aligned}
$$


Now we define

$$
M_{\mu}(t)=\sup _{0 \leq s \leq t}(1+s)^{2 \mu+2} E\left(\partial_{t}^{\mu} v(s)\right)
$$

Integrating (63) over $[0, t]$ and using (36) for $i=v$, we obtain

$$
\begin{aligned}
(1+ & t)^{2 \mu+2} E\left(\partial_{t}^{\mu} v(t)\right)+\int_{0}^{t}(1+s)^{2 \mu+2}\left\langle\partial_{t}^{\mu+1} v(s), B_{\lambda} \partial_{t}^{\mu+1} v(s)\right\rangle d s \\
\leq & E\left(\partial_{t}^{\mu} v(0)\right)+C \int_{0}^{t}(1+s)^{2 \mu+1} E_{L-\mu}\left(\partial_{t}^{\mu} v(s)\right) d s \\
& +C \int_{0}^{t}(1+s)^{2 \mu+2} E^{1 / 2}\left(\partial_{t}^{\mu} v(s)\right) \sum_{v=0}^{\mu} E_{L-v}^{1 / 2}\left(\partial_{t}^{v} v(s)\right) E_{L-(\mu-v)}^{1 / 2}\left(\partial_{t}^{\mu-v} v(s)\right) d s \\
\leq & E\left(\partial_{t}^{\mu} v(0)\right)+C \int_{0}^{t}(1+s)^{2 \mu+1} E_{L-\mu}\left(\partial_{t}^{\mu} v(s)\right) d s+\frac{C}{2}\left(M_{\mu}(t)\right)^{1 / 2} \\
& \times \sum_{v=0}^{\mu}\left\{\int_{0}^{t}(1+s)^{2 v+1} E_{L-v}\left(\partial_{t}^{v} v(s)\right) d s\right. \\
& \left.+\int_{0}^{t}(1+s)^{2(\mu-v)+1} E_{L-(\mu-v)}\left(\partial_{t}^{\mu-v} v(s)\right) d s\right\} \\
\leq & E\left(\partial_{t}^{\mu} v(0)\right)+C E_{0}+2(\mu+1) C E_{0}\left(M_{\mu}(t)\right)^{1 / 2} .
\end{aligned}
$$

From (64), we obtain

$$
\begin{aligned}
& (1+t)^{2 \mu+2} E\left(\partial_{t}^{\mu} v(t)\right)+\int_{0}^{t}(1+s)^{2 \mu+2}\left\langle\partial_{t}^{\mu+1} v(s), B_{\lambda} \partial_{t}^{\mu+1} v(s)\right\rangle d s \\
& \leq E_{0}+E_{0}\left(M_{\mu}(t)\right)^{1 / 2}
\end{aligned}
$$

Thus we have

$$
M_{\mu}(t) \leq E_{0}+E_{0}\left(M_{\mu}(t)\right)^{1 / 2} .
$$

It means that $M_{\mu}(t)$ is bounded in $[0, \infty)$. Thus it holds from (65) that

$$
(1+t)^{2 \mu+2} E\left(\partial_{t}^{\mu} v(t)\right)+\int_{0}^{t}(1+s)^{2 \mu+2}\left\langle\partial_{t}^{\mu+1} v(s), B_{\lambda} \partial_{t}^{\mu+1} v(s)\right\rangle d s \leq E_{0} .
$$

This means (37) for $i=\mu$. This completes the proof of theorem 4.1.

\section{Proof of lemma 3.1}

We prove lemma 3.1. First we prepare the estimates of the nonlinear term. 
Lemma 5.1 (Estimates of the nonlinear term). Let $L_{0} \leq \bar{L} \leq L, 0 \leq \mu \leq$ $\bar{L}-L_{0}$. Then there exists a constant $C>0$ such that for any $T, \delta>0$, $0<\lambda \leq 1$ and the local solution $v \in X_{\delta, T}$ to $(\mathrm{DW})_{\lambda}$ satisfy

$$
\begin{gathered}
\sum_{|a| \leq \bar{L}-\mu-1} \tilde{N}\left[\partial_{t}^{\mu} \nabla^{a} v, \partial_{t}^{\mu} \nabla^{a} v, \partial_{t} v\right](t) \leq C D_{\bar{L}, \mu}(v(t)), \\
\sum_{|a| \leq \bar{L}-\mu-1} \sum_{v=0}^{\mu-1}\left(\begin{array}{c}
\mu \\
v
\end{array}\right)\left\langle\partial_{t}^{\mu+1} \nabla^{a} v(t), N\left[\partial_{t}^{v} \nabla^{a} v, \partial_{t}^{\mu-v} v\right](t)\right\rangle \leq C D_{\bar{L}, \mu}(v(t)), \\
\sum_{|a| \leq \bar{L}-\mu-1} \sum_{\substack{b+c=a \\
b, c \neq 0}}\left\langle\partial_{t}^{\mu+1} \nabla^{a} v(t), \partial_{t}^{\mu} N\left[\nabla^{b} v, \nabla^{c} v\right](t)\right\rangle \leq C D_{\bar{L}, \mu}(v(t)), \\
\sum_{|a| \leq \bar{L}-\mu-1} \sum_{b+c=a}\left(\begin{array}{l}
a \\
b
\end{array}\right)\left\langle\partial_{t}^{\mu} \nabla^{a} v(t), \partial_{t}^{\mu} N\left[\nabla^{b} v, \nabla^{c} v\right](t)\right\rangle \leq C D_{\bar{L}, \mu}(v(t))
\end{gathered}
$$

and

$$
\sum_{|a| \leq \bar{L}-\mu-1}\left\langle\partial_{t}^{\mu} \nabla^{a} N[v, v](t),\left[h ; \nabla \partial_{t}^{\mu} \nabla^{a} v(t)\right]\right\rangle \leq \frac{C}{\lambda} D_{\bar{L}, \mu}(v(t))
$$

where $\tilde{N}$ and $D_{\bar{L}, \mu}$ are defined by (11) and (19).

Proof. First, we prove (66). Using lemma 2.1, we have

$$
\begin{aligned}
& \sum_{|a| \leq \bar{L}-\mu-1} \tilde{N}\left[\partial_{t}^{\mu} \nabla^{a} v, \partial_{t}^{\mu} \nabla^{a} v, \partial_{t} v\right](t) \\
& \quad \leq C \sum_{|a| \leq \bar{L}-\mu-1}\left\|\nabla \partial_{t}^{\mu} \nabla^{a} v(t)\right\|_{2}\left\|\nabla \partial_{t}^{\mu} \nabla^{a} v(t)\right\|_{2}\left\|\nabla \partial_{t} v(t)\right\|_{\infty} \\
& \quad \leq C \sum_{|a| \leq \bar{L}-\mu-1}\left\|\nabla \partial_{t}^{\mu} \nabla^{a} v(t)\right\|_{2}\left\|\nabla \partial_{t}^{\mu} \nabla^{a} v(t)\right\|_{2}\left\|\partial_{t} v(t)\right\|_{H^{[d / 2]+2}} \\
& \leq C E_{\bar{L}-\mu}^{1 / 2}\left(\partial_{t}^{\mu} v(t)\right) E_{\bar{L}-\mu}^{1 / 2}\left(\partial_{t}^{\mu} v(t)\right) E_{L_{0}}^{1 / 2}(v(t)) \\
& \leq C E_{\bar{L}-\mu}^{1 / 2}\left(\partial_{t}^{\mu} v(t)\right) \sum_{v=0}^{\mu} E_{\bar{L}-v}^{1 / 2}\left(\partial_{t}^{v} v(t)\right) E_{L_{0}}^{1 / 2}\left(\partial_{t}^{\mu-v} v(t)\right)=C D_{\bar{L}, \mu}(v(t)) .
\end{aligned}
$$

Next, we prove (67). We remark that if $|a| \leq \bar{L}-\mu-1$ and $0 \leq v \leq \mu-1$ then $|a|+1 \leq \bar{L}-v-1$. Hence it follows that 


$$
\begin{aligned}
\sum_{|a| \leq \bar{L}-\mu-1} & \sum_{v=0}^{\mu-1}\left(\begin{array}{c}
\mu \\
v
\end{array}\right)\left\langle\partial_{t}^{\mu+1} \nabla^{a} v(t), N\left[\partial_{t}^{v} \nabla^{a} v, \partial_{t}^{\mu-v} v\right](t)\right\rangle \\
\leq & C \sum_{|a| \leq \bar{L}-\mu-1}\left\|\partial_{t}^{\mu+1} \nabla^{a} v(t)\right\|_{2} \sum_{v=0}^{\mu-1} \sum_{j, k, l, m, n=1}^{d}\left\|\partial_{l}\left(\partial_{m} \partial_{t}^{v} \nabla^{a} v^{j}(t) \partial_{n} \partial_{t}^{\mu-v} v^{k}(t)\right)\right\|_{2} \\
\leq & C \sum_{|a| \leq \bar{L}-\mu-1}\left\|\partial_{t}^{\mu+1} \nabla^{a} v(t)\right\|_{2} \\
& \times \sum_{v=0}^{\mu-1} \sum_{j, k, l, m, n=1}^{d}\left\{\left\|\partial_{l} \partial_{m} \partial_{t}^{v} \nabla^{a} v^{j}(t)\right\|_{2}\left\|\partial_{n} \partial_{t}^{\mu-v} v^{k}(t)\right\|_{\infty}\right. \\
\leq & C E_{\bar{L}-\mu}^{1 / 2}\left(\partial_{t}^{\mu} v(t)\right) \sum_{v=0}^{\mu-1}\left\{E_{\bar{L}-v}^{1 / 2}\left(\partial_{t}^{v} v(t)\right) E_{L_{0}}^{1 / 2}\left(\partial_{t}^{\mu-v} v(t)\right)\right. \\
\leq & C E_{\bar{L}-\mu}^{1 / 2}\left(\partial_{t}^{\mu} v(t)\right) \sum_{v=0}^{\mu} E_{\bar{L}-v}^{1 / 2}\left(\partial_{t}^{v} v(t)\right) E_{L_{0}}^{1 / 2}\left(\partial_{t}^{\mu-v} v(t)\right)=C D_{\bar{L}, \mu}(v(t))
\end{aligned}
$$

Next, we prove (68). For any $|a| \leq \bar{L}-\mu-1, b+c=a$ and $b, c \neq 0$ it hold that

$$
\left\|\partial_{t}^{\mu} N\left[\nabla^{b} v, \nabla^{c} v\right](t)\right\|_{2} \leq C \sum_{v=0}^{\mu} E_{L_{0}}^{1 / 2}\left(\partial_{t}^{\mu-v} v(t)\right) E_{\bar{L}-v}^{1 / 2}\left(\partial_{t}^{v} v(t)\right)
$$

Because if $|a| \leq \bar{L}-\mu-1, b+c=a$ and $b, c \neq 0$ then we can decompose $c$ of the form: $c=c^{\prime}+c^{\prime \prime},\left|c^{\prime}\right|=|c|-1,\left|c^{\prime \prime}\right|=1$. Then using lemma 2.1 and lemma 2.2 we obtain

$$
\begin{gathered}
\left\|\partial_{t}^{\mu}\left(\partial_{l} \partial_{m} \nabla^{b} v^{i}(t) \partial_{n} \nabla^{c} v^{k}(t)\right)\right\|_{2} \\
\leq C \sum_{v=0}^{\mu}\left\|\partial_{t}^{v} \partial_{l} \partial_{m} \nabla^{b} v^{j}(t) \partial_{t}^{\mu-v} \partial_{n} \nabla^{c^{\prime \prime}} \nabla^{c^{\prime}} v^{k}(t)\right\|_{2} \\
\leq C \sum_{v=0}^{\mu}\left\{\left\|\nabla^{2} \partial_{t}^{v} v(t)\right\|_{\infty}\left\|\nabla^{2} \nabla^{b+c^{\prime}} \partial_{t}^{\mu-v} v(t)\right\|_{2}\right. \\
\left.\quad+\left\|\nabla^{2} \nabla^{b+c^{\prime}} \partial_{t}^{v} v(t)\right\|_{2}\left\|\nabla^{2} \partial_{t}^{\mu-v} v(t)\right\|_{\infty}\right\}
\end{gathered}
$$




$$
\begin{gathered}
\leq C \sum_{v=0}^{\mu}\left\{\left\|\nabla^{2} \partial_{t}^{v} v(t)\right\|_{H^{[d / 2]+1}}\left\|\nabla^{2} \nabla^{b+c^{\prime}} \partial_{t}^{\mu-v} v(t)\right\|_{2}\right. \\
\left.\quad+\left\|\nabla^{2} \nabla^{b+c^{\prime}} \partial_{t}^{v} v(t)\right\|_{2}\left\|\nabla^{2} \partial_{t}^{\mu-v} v(t)\right\|_{H^{[d / 2]+1}}\right\} \\
\leq C \sum_{v=0}^{\mu}\left\|\nabla^{2} \partial_{t}^{\mu-v} v(t)\right\|_{H^{[d / 2]+1}}\left\|\nabla^{2} \nabla^{b+c^{\prime}} \partial_{t}^{v} v(t)\right\|_{2} \\
\leq C \sum_{v=0}^{\mu} E_{L_{0}}^{1 / 2}\left(\partial_{t}^{\mu-v} v(t)\right) E_{\bar{L}-v}^{1 / 2}\left(\partial_{t}^{v} v(t)\right) .
\end{gathered}
$$

Similarly we obtain

$$
\left\|\partial_{t}^{\mu}\left(\partial_{m} \nabla^{b} v^{i} \partial_{l} \partial_{n} \nabla^{c} v^{k}\right)\right\|_{2} \leq C \sum_{v=0}^{\mu} E_{L_{0}}^{1 / 2}\left(\partial_{t}^{\mu-v} v(t)\right) E_{\bar{L}-v}^{1 / 2}\left(\partial_{t}^{v} v(t)\right) .
$$

Then we get (71). It follows from (71) that

$$
\begin{aligned}
& \sum_{\substack {|a| \leq \bar{L}-\mu-1 \\
\begin{subarray}{c}{b+c=a \\
b, c \neq 0{ | a | \leq \overline { L } - \mu - 1 \\
\begin{subarray} { c } { b + c = a \\
b , c \neq 0 } }\end{subarray}}\left\langle\partial_{t}^{\mu+1} \nabla^{a} v(t), \partial_{t}^{\mu} N\left[\nabla^{b} v, \nabla^{c} v\right](t)\right\rangle \\
& \leq \sum_{\substack {|a| \leq \bar{L}-\mu-1 \\
\begin{subarray}{c}{b+c \neq=a \\
b, c \neq 0{ | a | \leq \overline { L } - \mu - 1 \\
\begin{subarray} { c } { b + c \neq = a \\
b , c \neq 0 } }\end{subarray}}\left\|\partial_{t} \partial_{t}^{\mu} \nabla^{a} v(t)\right\|_{2}\left\|\partial_{t}^{\mu} N\left[\nabla^{b} v, \nabla^{c} v\right](t)\right\|_{2} \\
& \leq C E_{\bar{L}-\mu}^{1 / 2}\left(\partial_{t}^{\mu} v(t)\right) \sum_{v=0}^{\mu} E_{L_{0}}^{1 / 2}\left(\partial_{t}^{\mu-v} v(t)\right) E_{\bar{L}-v}^{1 / 2}\left(\partial_{t}^{v} v(t)\right)=C D_{\bar{L}, \mu}(v(t)) .
\end{aligned}
$$

Next, we prove (69). Using lemma 2.1 and lemma 2.2 we have

$$
\begin{aligned}
& \sum_{|a| \leq \bar{L}-\mu-1} \sum_{b+c=a}\left(\begin{array}{l}
a \\
b
\end{array}\right)\left\langle\partial_{t}^{\mu} \nabla^{a} v(t), \partial_{t}^{\mu} N\left[\nabla^{b} v, \nabla^{c} v\right](t)\right\rangle \\
& \leq C \sum_{|a| \leq \bar{L}-\mu-1} \sum_{b+c=a}\left|\sum_{i, j, k, l, m, n=1}^{d} \int_{\boldsymbol{R}^{d}} \partial_{t}^{\mu} \nabla^{a} v^{i} \partial_{t}^{\mu} \partial_{l}\left(\partial_{m} \nabla^{b} v^{j} \partial_{n} \nabla^{c} v^{k}\right) d x\right| \\
& =C \sum_{|a| \leq \bar{L}-\mu-1} \sum_{b+c=a}\left|\sum_{i, j, k, l, m, n=1}^{d} \int_{\boldsymbol{R}^{d}} \partial_{l} \partial_{t}^{\mu} \nabla^{a} v^{i} \partial_{t}^{\mu}\left(\partial_{m} \nabla^{b} v^{j} \partial_{n} \nabla^{c} v^{k}\right) d x\right| \\
& \leq C \sum_{|a| \leq \bar{L}-\mu-1}\left\|\nabla \partial_{t}^{\mu} \nabla^{a} v(t)\right\|_{2} \\
& \quad \times \sum_{v=0}^{\mu}\left\{\left\|\nabla \partial_{t}^{\mu-v} v(t)\right\|_{\infty}\left\|\nabla \nabla^{a} \partial_{t}^{v} v(t)\right\|_{2}+\left\|\nabla \nabla^{a} \partial_{t}^{\mu-v} v(t)\right\|_{2}\left\|\nabla \partial_{t}^{v} v(t)\right\|_{\infty}\right\}
\end{aligned}
$$




$$
\begin{aligned}
& \leq C \sum_{|a| \leq \bar{L}-\mu-1}\left\|\nabla \partial_{t}^{\mu} \nabla^{a} v(t)\right\|_{2} \sum_{v=0}^{\mu}\left\|\nabla \partial_{t}^{\mu-v} v(t)\right\|_{H^{[d / 2]+1}}\left\|\nabla \nabla^{a} \partial_{t}^{v} v(t)\right\|_{2} \\
& \leq C E_{\bar{L}-\mu}^{1 / 2}\left(\partial_{t}^{\mu} v(t)\right) \sum_{v=0}^{\mu} E_{L_{0}}^{1 / 2}\left(\partial_{t}^{\mu-v} v(t)\right) E_{\bar{L}-\mu}^{1 / 2}\left(\partial_{t}^{v} v\right) \leq C D_{\bar{L}, \mu}(v(t)) .
\end{aligned}
$$

Finally, we prove (70).

$$
\begin{aligned}
& \sum_{|a| \leq \bar{L}-\mu-1}\left\langle\partial_{t}^{\mu} \nabla^{a} N[v, v],\left[h ; \nabla \partial_{t}^{\mu} \nabla^{a} v\right]\right\rangle \\
& =\sum_{|a| \leq \bar{L}-\mu-1} \sum_{b+c=a}\left(\begin{array}{l}
a \\
b
\end{array}\right)\left\langle\partial_{t}^{\mu} N\left[\nabla^{b} v, \nabla^{c} v\right],\left[h ; \nabla \partial_{t}^{\mu} \nabla^{a} v\right]\right\rangle \\
& =\sum_{|a| \leq \bar{L}-\mu-1} \sum_{\substack{b+c=a \\
b, c \neq 0}}\left(\begin{array}{l}
a \\
b
\end{array}\right)\left\langle\partial_{t}^{\mu} N\left[\nabla^{b} v, \nabla^{c} v\right],\left[h ; \nabla \partial_{t}^{\mu} \nabla^{a} v\right]\right\rangle \\
& +2 \sum_{|a| \leq \bar{L}-\mu-1} \sum_{i, j, k, l, m, n=1}^{d} N_{l m n}^{i j k} \sum_{p=1}^{d} \int_{\boldsymbol{R}^{d}} \partial_{t}^{\mu} \partial_{l}\left(\partial_{m} \nabla^{a} v^{j} \partial_{n} v^{k}\right) h^{p} \partial_{p} \partial_{t}^{\mu} \nabla^{a} v^{i} d x \\
& =\sum_{|a| \leq \bar{L}-\mu-1} \sum_{\substack{b+c=a \\
b, c \neq 0}}\left(\begin{array}{l}
a \\
b
\end{array}\right)\left\langle\partial_{t}^{\mu} N\left[\nabla^{b} v, \nabla^{c} v\right],\left[h ; \nabla \partial_{t}^{\mu} \nabla^{a} v\right]\right\rangle \\
& +2 \sum_{|a| \leq \bar{L}-\mu-1} \sum_{i, j, k, l, m, n=1}^{d} N_{l m n}^{i j k} \\
& \times \sum_{p=1}^{d} \sum_{v=0}^{\mu-1}\left(\begin{array}{c}
\mu \\
v
\end{array}\right) \int_{\boldsymbol{R}^{d}} \partial_{l}\left(\partial_{m} \partial_{t}^{v} \nabla^{a} v^{j} \partial_{n} \partial_{t}^{\mu-v} v^{k}\right) h^{p} \partial_{p} \partial_{t}^{\mu} \nabla^{a} v^{i} d x \\
& +2 \sum_{|a| \leq \bar{L}-\mu-1} \sum_{i, j, k, l, m, n=1}^{d} N_{l m n}^{i j k} \sum_{p=1}^{d} \int_{\boldsymbol{R}^{d}} \partial_{l}\left(\partial_{m} \partial_{t}^{\mu} \nabla^{a} v^{j} \partial_{n} v^{k}\right) h^{p} \partial_{p} \partial_{t}^{\mu} \nabla^{a} v^{i} d x \\
& =\sum_{|a| \leq \bar{L}-\mu-1} \sum_{\substack{b+c=a \\
b, c \neq 0}}\left(\begin{array}{l}
a \\
b
\end{array}\right)\left\langle\partial_{t}^{\mu} N\left[\nabla^{b} v, \nabla^{c} v\right],\left[h ; \nabla \partial_{t}^{\mu} \nabla^{a} v\right]\right\rangle \\
& +2 \sum_{|a| \leq \bar{L}-\mu-1} \sum_{i, j, k, l, m, n=1}^{d} N_{l m n}^{i j k} \\
& \times \sum_{p=1}^{d} \sum_{v=0}^{\mu-1}\left(\begin{array}{c}
\mu \\
v
\end{array}\right) \int_{\boldsymbol{R}^{d}} \partial_{l}\left(\partial_{m} \partial_{t}^{v} \nabla^{a} v^{j} \partial_{n} \partial_{t}^{\mu-v} v^{k}\right) h^{p} \partial_{p} \partial_{t}^{\mu} \nabla^{a} v^{i} d x
\end{aligned}
$$




$$
\begin{aligned}
& -2 \sum_{|a| \leq \bar{L}-\mu-1} \sum_{i, j, k, l, m, n=1}^{d} N_{l m n}^{i j k} \sum_{p=1}^{d} \int_{\boldsymbol{R}^{d}} \partial_{m} \partial_{t}^{\mu} \nabla^{a} v^{j} \partial_{n} v^{k} \partial_{l} h^{p} \partial_{p} \partial_{t}^{\mu} \nabla^{a} v^{i} d x \\
& -2 \sum_{|a| \leq \bar{L}-\mu-1} \sum_{i, j, k, l, m, n=1}^{d} N_{l m n}^{i j k} \sum_{p=1}^{d} \int_{\boldsymbol{R}^{d}} \partial_{m} \partial_{t}^{\mu} \nabla^{a} v^{j} \partial_{n} v^{k} h^{p} \partial_{l} \partial_{p} \partial_{t}^{\mu} \nabla^{a} v^{i} d x \\
& =\sum_{|a| \leq \bar{L}-\mu-1} \sum_{\substack{b+c=a \\
b, c \neq 0}}\left(\begin{array}{l}
a \\
b
\end{array}\right)\left\langle\partial_{t}^{\mu} N\left[\nabla^{b} v, \nabla^{c} v\right],\left[h ; \nabla \partial_{t}^{\mu} \nabla^{a} v\right]\right\rangle \\
& +2 \sum_{|a| \leq \bar{L}-\mu-1} \sum_{i, j, k, l, m, n=1}^{d} N_{l m n}^{i j k} \\
& \times \sum_{p=1}^{d} \sum_{v=0}^{\mu-1}\left(\begin{array}{c}
\mu \\
v
\end{array}\right) \int_{\boldsymbol{R}^{d}} \partial_{l}\left(\partial_{m} \partial_{t}^{v} \nabla^{a} v^{j} \partial_{n} \partial_{t}^{\mu-v} v^{k}\right) h^{p} \partial_{p} \partial_{t}^{\mu} \nabla^{a} v^{i} d x \\
& -2 \sum_{|a| \leq \bar{L}-\mu-1} \sum_{i, j, k, l, m, n=1}^{d} N_{l m n}^{i j k} \sum_{p=1}^{d} \int_{\boldsymbol{R}^{d}} \partial_{m} \partial_{t}^{\mu} \nabla^{a} v^{j} \partial_{n} v^{k} \partial_{l} h^{p} \partial_{p} \partial_{t}^{\mu} \nabla^{a} v^{i} d x \\
& -\sum_{|a| \leq \bar{L}-\mu-1} \sum_{i, j, k, l, m, n=1}^{d} N_{l m n}^{i j k} \sum_{p=1}^{d} \int_{\boldsymbol{R}^{d}} \partial_{p}\left(\partial_{m} \partial_{t}^{\mu} \nabla^{a} v^{j} \partial_{l} \partial_{t}^{\mu} \nabla^{a} v^{i}\right) \partial_{n} v^{k} h^{p} d x \\
& =J_{1}+J_{2}+J_{3}+J_{4} \text {, }
\end{aligned}
$$

where we use (N1). For $J_{2}$ and $J_{3}$, it follows from (23) that

$$
\begin{aligned}
\left|J_{2}\right| \leq & C \sum_{|a| \leq \bar{L}-\mu-1} \sum_{i, j, k, l, m, n=1}^{d} \sum_{p=1}^{d} \sum_{v=0}^{\mu-1}\left\|\partial_{l}\left(\partial_{m} \partial_{t}^{v} \nabla^{a} v^{j} \partial_{n} \partial_{t}^{\mu-v} v^{k}\right) h^{p} \partial_{p} \partial_{t}^{\mu} \nabla^{a} v^{i}\right\|_{1} \\
\leq & C \sum_{|a| \leq \bar{L}-\mu-1} \sum_{p=1}^{d} \sum_{v=0}^{\mu-1}\left\|\nabla^{2} \partial_{t}^{v} \nabla^{a} v\right\|_{2}\left\|\nabla \partial_{t}^{\mu-v} v\right\|_{\infty}\left\|h^{p}\right\|_{\infty}\left\|\partial_{p} \partial_{t}^{\mu} \nabla^{a} v\right\|_{2} \\
& +C \sum_{|a| \leq \bar{L}-\mu-1} \sum_{p=1}^{d} \sum_{v=0}^{\mu-1}\left\|\nabla \partial_{t}^{v} \nabla^{a} v\right\|_{2}\left\|\nabla^{2} \partial_{t}^{\mu-v} v\right\|_{\infty}\left\|h^{p}\right\|_{\infty}\left\|\partial_{p} \partial_{t}^{\mu} \nabla^{a} v\right\|_{2} \\
\leq & C\|h\|_{\infty} \sum_{v=0}^{\mu-1} E_{\bar{L}-v}^{1 / 2}\left(\partial_{t}^{v} v(t)\right) E_{L_{0}}^{1 / 2}\left(\partial_{t}^{\mu-v} v(t)\right) E_{\bar{L}-\mu}^{1 / 2}\left(\partial_{t}^{\mu} v(t)\right) \leq \frac{C}{\lambda} D_{\bar{L}, \mu}(v(t))
\end{aligned}
$$

and 


$$
\begin{aligned}
\left|J_{3}\right| & \leq C \sum_{|a| \leq \bar{L}-\mu-1} \sum_{i, j, k, l, m, n=1}^{d} \sum_{p=1}^{d}\left\|\partial_{m} \partial_{t}^{\mu} \nabla^{a} v^{j} \partial_{n} v^{k} \partial_{l} h^{p} \partial_{p} \partial_{t}^{\mu} \nabla^{a} v^{i}\right\|_{1} \\
& \leq C \sum_{|a| \leq \bar{L}-\mu-1}\left\|\nabla \partial_{t}^{\mu} \nabla^{a} v\right\|_{2}\|\nabla v\|_{\infty}\|\nabla h\|_{\infty}\left\|\nabla \partial_{t}^{\mu} \nabla^{a} v\right\|_{2} \\
& \leq C E_{\bar{L}-\mu}^{1 / 2}\left(\partial_{t}^{\mu} v(t)\right) E_{\bar{L}-\mu}^{1 / 2}\left(\partial_{t}^{\mu} v(t)\right) E_{L_{0}}^{1 / 2}(v(t)) \leq C D_{\mu}(v(t)) \leq \frac{C}{\lambda} D_{\bar{L}, \mu}(v(t)) .
\end{aligned}
$$

From (23) and (71), we get

$$
\begin{aligned}
\left|J_{1}\right| & \leq C \sum_{|a| \leq \bar{L}-\mu-1} \sum_{\substack{b+c=a \\
b, c \neq 0}}\left|\left\langle\partial_{t}^{\mu} N\left[\nabla^{b} v, \nabla^{c} v\right],\left[h ; \nabla \partial_{t}^{\mu} \nabla^{a} v\right]\right\rangle\right| \\
& \leq C \sum_{|a| \leq \bar{L}-\mu-1} \sum_{\substack{b+c=a \\
b, c \neq 0}} \|\left\langle\partial_{t}^{\mu} N\left[\nabla^{b} v, \nabla^{c} v\right]\left\|_{2}\right\| h\left\|_{\infty}\right\| \nabla \partial_{t}^{\mu} \nabla^{a} v \|_{2}\right. \\
& \leq C\|h\|_{\infty} E_{\bar{L}-\mu}^{1 / 2}\left(\partial_{t}^{\mu} v(t)\right) \sum_{v=0}^{\mu} E_{L_{0}}^{1 / 2}\left(\partial_{t}^{\mu-v} v(t)\right) E_{\bar{L}-v}^{1 / 2}\left(\partial_{t}^{v} v(t)\right) \leq \frac{C}{\lambda} D_{\bar{L}, \mu}(v(t)) .
\end{aligned}
$$

From (71), we also have

$$
\begin{aligned}
\left|J_{4}\right|= & \left|\sum_{|a| \leq \bar{L}-\mu-1} \sum_{i, j, k, l, m, n=1}^{d} N_{l m n}^{i j k} \sum_{p=1}^{d} \int_{\boldsymbol{R}^{d}} \partial_{p}\left(\partial_{m} \partial_{t}^{\mu} \nabla^{a} v^{j} \partial_{l} \partial_{t}^{\mu} \nabla^{a} v^{i}\right) \partial_{n} v^{k} h^{p} d x\right| \\
= & \left|\sum_{|a| \leq \bar{L}-\mu-1} \sum_{i, j, k, l, m, n=1}^{d} N_{l m n}^{i j k} \sum_{p=1}^{d} \int_{\boldsymbol{R}^{d}} \partial_{m} \partial_{t}^{\mu} \nabla^{a} v^{j} \partial_{l} \partial_{t}^{\mu} \nabla^{a} v^{i} \partial_{p}\left(\partial_{n} v^{k} h^{p}\right) d x\right| \\
\leq & C \sum_{|a| \leq \bar{L}-\mu-1} \sum_{i, j, k, l, m, n=1}^{d} \sum_{p=1}^{d} \int_{\boldsymbol{R}^{d}}\left|\partial_{m} \partial_{t}^{\mu} \nabla^{a} v^{j} \partial_{l} \partial_{t}^{\mu} \nabla^{a} v^{i} \partial_{p} \partial_{n} v^{k} h^{p}\right| d x \\
& +C \sum_{|a| \leq \bar{L}-\mu-1} \sum_{|a| \leq \bar{L}-\mu-1}^{d i, k, l, m, n=1} \sum_{p=1}^{d} \int_{\boldsymbol{R}^{d}}\left|\partial_{m} \partial_{t}^{\mu} \nabla^{a} v^{j} \partial_{l} \partial_{t}^{\mu} \nabla^{a} v^{i} \partial_{n} v^{k} \partial_{p} h^{p}\right| d x \\
\leq & C \sum_{t}^{\mu} \nabla^{a} v\left\|_{2}\right\| \nabla \partial_{t}^{\mu} \nabla^{a} v \|_{2}\left(\left\|\nabla^{2} v\right\|_{\infty}\|h\|_{\infty}+\|\nabla v\|_{\infty}\|\nabla h\|_{\infty}\right) \\
\leq & C\left(1+\frac{1}{\lambda}\right) E_{\bar{L}-\mu}^{1 / 2}\left(\partial_{t}^{\mu} v(t)\right) E_{\bar{L}-\mu}^{1 / 2}\left(\partial_{t}^{\mu} v(t)\right) E_{L_{0}}^{1 / 2}(v(t)) \leq \frac{C}{\lambda} D_{\bar{L}, \mu}(v(t)) .
\end{aligned}
$$

Hence we obtain (70), which completes the proof of lemma 5.1. 
Because of dissipation effect $(\mathbf{B 2})_{\lambda}$, we are expected to decrease the energy in $|x| \gg 1$. Next lemma corresponds to this phenomenon.

Lemma 5.2. Let $L_{0} \leq \bar{L} \leq L, 0 \leq \mu \leq \bar{L}-L_{0}$. Then there exists a constant $C>0$ such that for any $T, \delta>0,0<\lambda \leq 1$ and the local solution $v \in X_{\delta, T}$ to $(\mathrm{DW})_{\lambda}$ satisfy

$$
\begin{gathered}
\frac{d}{d t} \tilde{E}_{\bar{L}, \mu}(v(t))+\sum_{|a| \leq \bar{L}-\mu-1}\left\langle\partial_{t}^{\mu+1} \nabla^{a} v(t), B_{\lambda} \partial_{t}^{\mu+1} \nabla^{a} v(t)\right\rangle \\
\quad \leq C \lambda^{2} E_{\bar{L}-\mu}\left(\partial_{t}^{\mu} v(t)\right)+C D_{\bar{L}, \mu}(v(t))
\end{gathered}
$$

and

$$
\begin{aligned}
& \frac{d}{d t} \sum_{|a| \leq \bar{L}-\mu-1}\left\{\left\langle\partial_{t}^{\mu} \nabla^{a} v(t), \partial_{t}^{\mu+1} \nabla^{a} v(t)\right\rangle+\frac{1}{2}\left\langle\partial_{t}^{\mu} \nabla^{a} v(t), B_{\lambda} \partial_{t}^{\mu} \nabla^{a} v(t)\right\rangle\right\} \\
& \quad-\sum_{|a| \leq \bar{L}-\mu-1}\left\|\partial_{t}^{\mu+1} \nabla^{a} v(t)\right\|_{2}^{2}+\sum_{|a| \leq \bar{L}-\mu-1}\left\|\nabla \partial_{t}^{\mu} \nabla^{a} v(t)\right\|_{2}^{2} \\
& \leq C \lambda^{2} E_{\bar{L}-\mu}\left(\partial_{t}^{\mu} v(t)\right)+C D_{\bar{L}, \mu}(v(t)) .
\end{aligned}
$$

Proof. Let $L_{0} \leq \bar{L} \leq L$ and $0 \leq \mu \leq \bar{L}-L_{0}$. First we show (72). We calculate

$$
\frac{d}{d t} \tilde{E}_{\bar{L}, \mu}(v(t))=\sum_{|a| \leq \bar{L}-\mu-1} \frac{d}{d t} E\left(\partial_{t}^{\mu} \nabla^{a} v(t)\right)+\sum_{|a| \leq \bar{L}-\mu-1} \frac{d}{d t} \tilde{N}\left[\partial_{t}^{\mu} \nabla^{a} v, \partial_{t}^{\mu} \nabla^{a} v, v\right](t) .
$$

For the second terms of (74), for any $|a| \leq \bar{L}-\mu-1$, we need the following equality:

$$
\begin{aligned}
\left\langle\partial_{t}^{\mu+1} \nabla^{a} v, \partial_{t}^{\mu} N\left[\nabla^{a} v, v\right]\right\rangle & \\
= & \left\langle\partial_{t}^{\mu+1} \nabla^{a} v, N\left[\partial_{t}^{\mu} \nabla^{a} v, v\right]\right\rangle+\sum_{v=0}^{\mu-1}\left(\begin{array}{c}
\mu \\
v
\end{array}\right)\left\langle\partial_{t}^{\mu+1} \nabla^{a} v, N\left[\partial_{t}^{v} \nabla^{a} v, \partial_{t}^{\mu-v} v\right]\right\rangle \\
= & \sum_{i, j, k, l, m, n=1}^{d} N_{l m n}^{i j k} \int_{\boldsymbol{R}^{d}} \partial_{t}^{\mu+1} \nabla^{a} v^{i} \partial_{l}\left(\partial_{m} \partial_{t}^{\mu} \nabla^{a} v^{j} \partial_{n} v^{k}\right) d x \\
& +\sum_{v=0}^{\mu-1}\left(\begin{array}{c}
\mu \\
v
\end{array}\right)\left\langle\partial_{t}^{\mu+1} \nabla^{a} v, N\left[\partial_{t}^{v} \nabla^{a} v, \partial_{t}^{\mu-v} v\right]\right\rangle \\
= & -\sum_{i, j, k, l, m, n=1}^{d} N_{l m n}^{i j k} \int_{\boldsymbol{R}^{d}} \partial_{l} \partial_{t}^{\mu+1} \nabla^{a} v^{i} \partial_{m} \partial_{t}^{\mu} \nabla^{a} v^{j} \partial_{n} v^{k} d x
\end{aligned}
$$




$$
\begin{aligned}
& +\sum_{v=0}^{\mu-1}\left(\begin{array}{c}
\mu \\
v
\end{array}\right)\left\langle\partial_{t}^{\mu+1} \nabla^{a} v, N\left[\partial_{t}^{v} \nabla^{a} v, \partial_{t}^{\mu-v} v\right]\right\rangle \\
= & -\frac{1}{2} \frac{d}{d t} \sum_{i, j, k, l, m, n=1}^{d} N_{l m n}^{i j k} \int_{\boldsymbol{R}^{d}} \partial_{l} \partial_{t}^{\mu} \nabla^{a} v^{i} \partial_{m} \partial_{t}^{\mu} \nabla^{a} v^{j} \partial_{n} v^{k} d x \\
& +\frac{1}{2} \sum_{i, j, k, l, m, n=1}^{d} N_{l m n}^{i j k} \int_{\boldsymbol{R}^{d}} \partial_{l} \partial_{t}^{\mu} \nabla^{a} v^{i} \partial_{m} \partial_{t}^{\mu} \nabla^{a} v^{j} \partial_{n} \partial_{t} v^{k} d x \\
& +\sum_{v=0}^{\mu-1}\left(\begin{array}{c}
\mu \\
v
\end{array}\right)\left\langle\partial_{t}^{\mu+1} \nabla^{a} v, N\left[\partial_{t}^{v} \nabla^{a} v, \partial_{t}^{\mu-v} v\right]\right\rangle \\
= & -\frac{1}{2} \frac{d}{d t} \tilde{N}\left[\partial_{t}^{\mu} \nabla^{a} v, \partial_{t}^{\mu} \nabla^{a} v, v\right]+\frac{1}{2} \tilde{N}\left[\partial_{t}^{\mu} \nabla^{a} v, \partial_{t}^{\mu} \nabla^{a} v, \partial_{t} v\right] \\
& +\sum_{v=0}^{\mu-1}\left(\begin{array}{c}
\mu \\
v
\end{array}\right)\left\langle\partial_{t}^{\mu+1} \nabla^{a} v, N\left[\partial_{t}^{v} \nabla^{a} v, \partial_{t}^{\mu-v} v\right]\right\rangle,
\end{aligned}
$$

where we use (N1). To handle the first terms of (74), we apply $\partial_{t}^{\mu} \nabla^{a}$ to (DW) $)_{\lambda}$. We get

$$
\partial_{t}^{\mu+2} \nabla^{a} v-\triangle \partial_{t}^{\mu} \nabla^{a} v+\nabla^{a}\left(B_{\lambda} \partial_{t}^{\mu+1} v\right)=\sum_{b+c=a}\left(\begin{array}{l}
a \\
b
\end{array}\right) \partial_{t}^{\mu} N\left[\nabla^{b} v, \nabla^{c} v\right],
$$

which yields that

$$
\begin{aligned}
\sum_{|a| \leq \bar{L}-\mu-1} \frac{d}{d t} E\left(\partial_{t}^{\mu} \nabla^{a} v\right)= & -\sum_{|a| \leq \bar{L}-\mu-1}\left\langle\partial_{t}^{\mu+1} \nabla^{a} v, \nabla^{a}\left(B_{\lambda} \partial_{t}^{\mu+1} v\right)\right\rangle \\
& +\sum_{|a| \leq \bar{L}-\mu-1} \sum_{b+c=a}\left(\begin{array}{l}
a \\
b
\end{array}\right)\left\langle\partial_{t}^{\mu+1} \nabla^{a} v, \partial_{t}^{\mu} N\left[\nabla^{b} v, \nabla^{c} v\right]\right\rangle .
\end{aligned}
$$

Combining (74), (75), (77) and lemma 5.1, we get

$$
\begin{aligned}
\frac{d}{d t} \tilde{E}_{\bar{L}, \mu}\left(\partial_{t}^{\mu} v\right)= & \sum_{|a| \leq \bar{L}-\mu-1} \frac{d}{d t} E\left(\partial_{t}^{\mu} \nabla^{a} v\right)+\sum_{|a| \leq \bar{L}-\mu-1} \frac{d}{d t} \tilde{N}\left[\partial_{t}^{\mu} \nabla^{a} v, \partial_{t}^{\mu} \nabla^{a} v, v\right] \\
= & -\sum_{|a| \leq \bar{L}-\mu-1}\left\langle\partial_{t}^{\mu+1} \nabla^{a} v, \nabla^{a}\left(B_{\lambda} \partial_{t}^{\mu+1} v\right)\right\rangle \\
& +\sum_{|a| \leq \bar{L}-\mu-1} \sum_{b+c=a}\left(\begin{array}{l}
a \\
b
\end{array}\right)\left\langle\partial_{t}^{\mu+1} \nabla^{a} v, \partial_{t}^{\mu} N\left[\nabla^{b} v, \nabla^{c} v\right]\right\rangle
\end{aligned}
$$




$$
\begin{aligned}
& -2 \sum_{|a| \leq \bar{L}-\mu-1}\left\langle\partial_{t}^{\mu+1} \nabla^{a} v, \partial_{t}^{\mu} N\left[\nabla^{a} v, v\right]\right\rangle \\
& +\sum_{|a| \leq \bar{L}-\mu-1} \tilde{N}\left[\partial_{t}^{\mu} \nabla^{a} v, \partial_{t}^{\mu} \nabla^{a} v, \partial_{t} v\right] \\
& +2 \sum_{|a| \leq \bar{L}-\mu-1} \sum_{v=0}^{\mu-1}\left(\begin{array}{c}
\mu \\
v
\end{array}\right)\left\langle\partial_{t}^{\mu+1} \nabla^{a} v, N\left[\partial_{t}^{v} \nabla^{a} v, \partial_{t}^{\mu-v} v\right]\right\rangle \\
& =-\sum_{|a| \leq \bar{L}-\mu-1}\left\langle\partial_{t}^{\mu+1} \nabla^{a} v, \nabla^{a}\left(B_{\lambda} \partial_{t}^{\mu+1} v\right)\right\rangle \\
& +\sum_{|a| \leq \bar{L}-\mu-1} \sum_{b+c=a}^{b+c \neq 0}\left(\begin{array}{c}
a \\
b
\end{array}\right)\left\langle\partial_{t}^{\mu+1} \nabla^{a} v, \partial_{t}^{\mu} N\left[\nabla^{b} v, \nabla^{c} v\right]\right\rangle \\
& +\sum_{|a| \leq \bar{L}-\mu-1} \tilde{N}\left[\partial_{t}^{\mu} \nabla^{a} v, \partial_{t}^{\mu} \nabla^{a} v, \partial_{t} v\right] \\
& +2 \sum_{|a| \leq \bar{L}-\mu-1} \sum_{v=0}^{\mu-1}\left(\begin{array}{c}
\mu \\
v
\end{array}\right)\left\langle\partial_{t}^{\mu+1} \nabla^{a} v, N\left[\partial_{t}^{v} \nabla^{a} v, \partial_{t}^{\mu-v} v\right]\right\rangle \\
& \leq-\sum_{|a| \leq \bar{L}-\mu-1}\left\langle\partial_{t}^{\mu+1} \nabla^{a} v, \nabla^{a}\left(B_{\lambda} \partial_{t}^{\mu+1} v\right)\right\rangle+C D_{\bar{L}, \mu}(v) .
\end{aligned}
$$

Since for $\lambda \leq 1$, we have

$$
\begin{aligned}
& -\sum_{|a| \leq \bar{L}-\mu-1}\left\langle\partial_{t}^{\mu+1} \nabla^{a} v, \nabla^{a}\left(B_{\lambda} \partial_{t}^{\mu+1} v\right)\right\rangle \\
& =-\sum_{|a| \leq \bar{L}-\mu-1}\left\langle\partial_{t}^{\mu+1} \nabla^{a} v, B_{\lambda} \partial_{t}^{\mu+1} \nabla^{a} v\right\rangle \\
& \quad-\sum_{|a| \leq \bar{L}-\mu-1} \sum_{\substack{b \leq a \\
b \neq 0}}\left(\begin{array}{l}
a \\
b
\end{array}\right)\left\langle\partial_{t}^{\mu+1} \nabla^{a} v, \nabla^{b} B_{\lambda}(x) \partial_{t}^{\mu+1} \nabla^{a-b} v\right\rangle \\
& \leq-\sum_{|a| \leq \bar{L}-\mu-1}\left\langle\partial_{t}^{\mu+1} \nabla^{a} v, B_{\lambda} \partial_{t}^{\mu+1} \nabla^{a} v\right\rangle \\
& \quad+C \sum_{|a| \leq \bar{L}-\mu-1} \sum_{\substack{b \leq a \\
b \neq 0}}\left\|\nabla^{b} B_{\lambda}\right\|_{\infty}\left\|\partial_{t}^{\mu+1} \nabla^{a} v\right\|_{2}\left\|\partial_{t}^{\mu+1} \nabla^{a-b} v\right\|_{2} \\
& \leq-\sum_{|a| \leq \bar{L}-\mu-1}\left\langle\partial_{t}^{\mu+1} \nabla^{a} v, B_{\lambda} \partial_{t}^{\mu+1} \nabla^{a} v\right\rangle+C \lambda^{2} E_{\bar{L}-\mu}\left(\partial_{t}^{\mu} v\right) .
\end{aligned}
$$

Thus we obtain (72). 
Next we prove (73). Using (76) and lemma 5.1 (69), we have

$$
\begin{aligned}
& \frac{d}{d t}\left\{\sum_{|a| \leq \bar{L}-\mu-1}\left\langle\partial_{t}^{\mu} \nabla^{a} v, \partial_{t}^{\mu+1} \nabla^{a} v\right\rangle+\frac{1}{2} \sum_{|a| \leq \bar{L}-\mu-1}\left\langle\partial_{t}^{\mu} \nabla^{a} v, B_{\lambda} \partial_{t}^{\mu} \nabla^{a} v\right\rangle\right\} \\
& =\sum_{|a| \leq \bar{L}-\mu-1}\left\{\left\|\partial_{t}^{\mu+1} \nabla^{a} v\right\|_{2}^{2}+\left\langle\partial_{t}^{\mu} \nabla^{a} v, \partial_{t}^{\mu+2} \nabla^{a} v\right\rangle+\left\langle\partial_{t}^{\mu} \nabla^{a} v, B_{\lambda} \partial_{t}^{\mu+1} \nabla^{a} v\right\rangle\right\} \\
& \leq \sum_{|a| \leq \bar{L}-\mu-1}\left\|\partial_{t}^{\mu+1} \nabla^{a} v\right\|_{2}^{2}-\sum_{|a| \leq \bar{L}-\mu-1}\left\|\nabla \partial_{t}^{\mu} \nabla^{a} v\right\|_{2}^{2} \\
& \quad-\sum_{\substack{b \leq a \\
b \neq 0}}\left(\begin{array}{l}
a \\
b
\end{array}\right)\left\langle\partial_{t}^{\mu} \nabla^{a} v, \nabla^{b} B_{\lambda} \partial_{t}^{\mu+1} \nabla^{a-b} v\right\rangle+C D_{\bar{L}, \mu}(v) .
\end{aligned}
$$

For $\lambda \leq 1$, we have

$$
\begin{aligned}
& \sum_{|a| \leq \bar{L}-\mu-1} \sum_{\substack{b \leq a \\
b \neq 0}}\left(\begin{array}{c}
a \\
b
\end{array}\right)\left|\left\langle\partial_{t}^{\mu} \nabla^{a} v, \nabla^{b} B_{\lambda} \partial_{t}^{\mu+1} \nabla^{a-b} v\right\rangle\right| \\
& =\sum_{1 \leq|a| \leq \bar{L}-\mu-1} \sum_{\substack{b \leq a \\
b \neq 0}}\left(\begin{array}{l}
a \\
b
\end{array}\right)\left|\left\langle\partial_{t}^{\mu} \nabla^{a} v, \nabla^{b} B_{\lambda} \partial_{t}^{\mu+1} \nabla^{a-b} v\right\rangle\right| \\
& \leq \sum_{1 \leq|a| \leq \bar{L}-\mu-1} \sum_{\substack{b \leq a \\
b \neq 0}}\left(\begin{array}{l}
a \\
b
\end{array}\right)\left\|\nabla^{b} B_{\lambda}\right\|_{\infty}\left\|\partial_{t}^{\mu} \nabla^{a} v\right\|_{2}\left\|\partial_{t}^{\mu+1} \nabla^{a-b} v\right\|_{2} \leq \lambda^{2} C E_{\bar{L}-\mu}\left(\partial_{t}^{\mu} v\right),
\end{aligned}
$$

which yields (73) from (79). This completes the proof of lemma 5.2.

We can't expect the effect of dissipation in $|x| \leq R / \lambda$ since $B_{\lambda}(x)$ may not strictly positive. So we use the local energy decay property in $(\mathrm{DW})_{\lambda}$. We lead the estimate which corresponding this property by using the argument of Nakao [11] and Ikehata [5].

Lemma 5.3. Let $L_{0} \leq \bar{L} \leq L, 0 \leq \mu \leq \bar{L}-L_{0}$. Then there exists a constant $C>0$ such that for any $K, T, \delta>0,0<\lambda \leq 1$ and the local solution $v \in X_{\delta, T}$ to $(\mathrm{DW})_{\lambda}$ satisfy

$$
\begin{aligned}
& \frac{d}{d t}\left\{\sum_{|a| \leq \bar{L}-\mu-1}\left\langle\partial_{t}^{\mu+1} \nabla^{a} v(t),\left[h ; \nabla \partial_{t}^{\mu} \nabla^{a} v(t)\right]\right\rangle\right\} \\
& \quad+\sum_{|a| \leq \bar{L}-\mu-1} \int_{\boldsymbol{R}^{d}}\left\{\frac{d \phi+|x| \phi^{\prime}}{2}\right\}\left|\partial_{t}^{\mu+1} \nabla^{a} v(t, x)\right|^{2} d x \\
& \quad+\sum_{|a| \leq \bar{L}-\mu-1} \int_{\boldsymbol{R}^{d}}\left\{\phi+|x| \phi^{\prime}-\frac{d \phi+|x| \phi^{\prime}}{2}\right\}\left|\nabla \partial_{t}^{\mu} \nabla^{a} v(t, x)\right|^{2} d x
\end{aligned}
$$




$$
\begin{aligned}
\leq & \lambda C E_{\bar{L}-\mu}\left(\partial_{t}^{\mu} v(t)\right)+\frac{C}{\lambda} D_{\bar{L}, \mu}(v(t)) \\
& +\frac{K}{4} \sum_{|a| \leq \bar{L}-\mu-1}\left\langle\partial_{t}^{\mu+1} \nabla^{a} v(t), B_{\lambda} \partial_{t}^{\mu+1} \nabla^{a} v(t)\right\rangle \\
& +\frac{2\|B\|_{\infty} b_{0}^{2} R^{2}}{\lambda K} E_{\bar{L}-\mu}\left(\partial_{t}^{\mu} v(t)\right),
\end{aligned}
$$

where $\phi=\phi(|x|), h$ are defined by (17).

Proof. Let $|a| \leq \bar{L}-\mu-1$. We apply $\partial_{t}^{\mu} \nabla^{a}$ to $(\mathrm{DW})_{\lambda}$ and take inner product the equation by $\left[h ; \nabla \partial_{t}^{\mu} \nabla^{a} v\right]$ we obtain

$$
\begin{aligned}
& \left\langle\partial_{t}^{\mu+2} \nabla^{a} v,\left[h ; \nabla \partial_{t}^{\mu} \nabla^{a} v\right]\right\rangle-\left\langle\Delta \partial_{t}^{\mu} \nabla^{a} v,\left[h ; \nabla \partial_{t}^{\mu} \nabla^{a} v\right]\right\rangle \\
& \quad+\left\langle\nabla^{a}\left(B_{\lambda} \partial_{t}^{\mu+1} v\right),\left[h ; \nabla \partial_{t}^{\mu} \nabla^{a} v\right]\right\rangle=\left\langle\partial_{t}^{\mu} \nabla^{a} N[v, v],\left[h ; \nabla \partial_{t}^{\mu} \nabla^{a} v\right]\right\rangle .
\end{aligned}
$$

Noting (81),

$$
\begin{aligned}
\left\langle\partial_{t}^{\mu+2}\right. & \left.\nabla^{a} v,\left[h ; \nabla \partial_{t}^{\mu} \nabla^{a} v\right]\right\rangle \\
= & \frac{d}{d t}\left\langle\partial_{t}^{\mu+1} \nabla^{a} v,\left[h ; \nabla \partial_{t}^{\mu} \nabla^{a} v\right]\right\rangle-\sum_{k=1}^{d} \sum_{i=1}^{d} \int_{\boldsymbol{R}^{d}} \partial_{t}^{\mu+1} \nabla^{a} v^{k} h^{i} \partial_{i} \partial_{t}^{\mu+1} \nabla^{a} v^{k} d x \\
= & \frac{d}{d t}\left\langle\partial_{t}^{\mu+1} \nabla^{a} v,\left[h ; \nabla \partial_{t}^{\mu} \nabla^{a} v\right]\right\rangle-\frac{1}{2} \sum_{k=1}^{d} \sum_{i=1}^{d} \int_{\boldsymbol{R}^{d}} \partial_{i}\left|\partial_{t}^{\mu+1} \nabla^{a} v^{k}\right|^{2} h^{i} d x \\
= & \frac{d}{d t}\left\langle\partial_{t}^{\mu+1} \nabla^{a} v,\left[h ; \nabla \partial_{t}^{\mu} \nabla^{a} v\right]\right\rangle+\frac{1}{2} \int_{\boldsymbol{R}^{d}}\left|\partial_{t}^{\mu+1} \nabla^{a} v\right|^{2} \operatorname{div} h d x
\end{aligned}
$$

and

$$
\begin{aligned}
-\langle\triangle & \left.\partial_{t}^{\mu} \nabla^{a} v(t),\left[h ; \nabla \partial_{t}^{\mu} \nabla^{a} v(t)\right]\right\rangle \\
& =\sum_{i, k=1}^{d} \int_{\boldsymbol{R}^{d}} \nabla \partial_{t}^{\mu} \nabla^{a} v^{k} \cdot \nabla\left(h^{i} \partial_{i} \partial_{t}^{\mu} \nabla^{a} v^{k}\right) d x \\
& =\sum_{i, k=1}^{d} \int_{\boldsymbol{R}^{d}} \nabla \partial_{t}^{\mu} \nabla^{a} v^{k} \cdot \nabla h^{i} \partial_{i} \partial_{t}^{\mu} \nabla^{a} v^{k} d x+\sum_{i, k=1}^{d} \int_{\boldsymbol{R}^{d}} \nabla \partial_{t}^{\mu} \nabla^{a} v^{k} \cdot \nabla \partial_{i} \partial_{t}^{\mu} \nabla^{a} v^{k} h^{i} d x \\
& =\sum_{i, j, k=1}^{d} \int_{\boldsymbol{R}^{d}} \partial_{j} \partial_{t}^{\mu} \nabla^{a} v^{k} \partial_{j} h^{i} \partial_{i} \partial_{t}^{\mu} \nabla^{a} v^{k} d x+\frac{1}{2} \sum_{i, k=1}^{d} \int_{\boldsymbol{R}^{d}} \partial_{i}\left|\nabla \partial_{t}^{\mu} \nabla^{a} v^{k}\right|^{2} h^{i} d x \\
& =\sum_{i, j, k=1}^{d} \int_{\boldsymbol{R}^{d}} \partial_{j} \partial_{t}^{\mu} \nabla^{a} v^{k} \partial_{j} h^{i} \partial_{i} \partial_{t}^{\mu} \nabla^{a} v^{k} d x-\frac{1}{2} \int_{\boldsymbol{R}^{d}}\left|\nabla \partial_{t}^{\mu} \nabla^{a} v\right|^{2} \operatorname{div} h d x,
\end{aligned}
$$


we obtain

(82) $\frac{d}{d t}\left\langle\partial_{t}^{\mu+1} \nabla^{a} v,\left[h ; \nabla \partial_{t}^{\mu} \nabla^{a} v\right]\right\rangle+\frac{1}{2} \int_{\boldsymbol{R}^{d}}\left|\partial_{t}^{\mu+1} \nabla^{a} v\right|^{2} \operatorname{div} h d x$

$$
\begin{aligned}
& -\frac{1}{2} \int_{\boldsymbol{R}^{d}}\left|\nabla \partial_{t}^{\mu} \nabla^{a} v\right|^{2} \operatorname{div} h d x+\sum_{i, j, k=1}^{d} \int_{\boldsymbol{R}^{d}} \partial_{j} \partial_{t}^{\mu} \nabla^{a} v^{k} \partial_{j} h^{i} \partial_{i} \partial_{t}^{\mu} \nabla^{a} v^{k} d x \\
= & -\left\langle\nabla^{a}\left(B_{\lambda} \partial_{t}^{\mu+1} v\right),\left[h ; \nabla \partial_{t}^{\mu} \nabla^{a} v\right]\right\rangle+\left\langle\partial_{t}^{\mu} \nabla^{a} N[v, v],\left[h ; \nabla \partial_{t}^{\mu} \nabla^{a} v\right]\right\rangle .
\end{aligned}
$$

Now we remark that it holds that

$$
\frac{\partial h^{i}}{\partial x_{j}}=\delta_{i j} \phi(|x|)+\phi^{\prime}(|x|) \frac{x_{i} x_{j}}{|x|}
$$

and

$$
\operatorname{div} h(x)=d \phi(|x|)+|x| \phi^{\prime}(|x|) \quad\left(x \in \boldsymbol{R}^{d}\right) .
$$

Furthermore using $\phi^{\prime}(r) \leq 0$ we get

$$
\begin{aligned}
& \sum_{i, j, k=1}^{d} \int_{\boldsymbol{R}^{d}} \partial_{j} \partial_{t}^{\mu} \nabla^{a} v^{k} \partial_{j} h^{i} \partial_{i} \partial_{t}^{\mu} \nabla^{a} v^{k} d x \\
& \quad=\sum_{i, k=1}^{d} \int_{\boldsymbol{R}^{d}} \partial_{i} \partial_{t}^{\mu} \nabla^{a} v^{k} \phi \partial_{t}^{\mu} \partial_{i} \nabla^{a} v^{k} d x+\sum_{i, j, k=1}^{d} \int_{\boldsymbol{R}^{d}} \partial_{j} \partial_{t}^{\mu} \nabla^{a} v^{k} \phi^{\prime} \frac{x_{i} x_{j}}{|x|} \partial_{i} \partial_{t}^{\mu} \nabla^{a} v^{k} d x \\
& \quad=\int_{\boldsymbol{R}^{d}}\left|\nabla \partial_{t}^{\mu} \nabla^{a} v\right|^{2} \phi d x+\sum_{k=1}^{d} \int_{\boldsymbol{R}^{d}}\left|x \cdot \nabla \partial_{t}^{\mu} \nabla^{a} v^{k}\right|^{2} \phi^{\prime} \frac{1}{|x|} d x \\
& \quad \geq \int_{\boldsymbol{R}^{d}}\left\{\phi+|x| \phi^{\prime}\right\}\left|\nabla \partial_{t}^{\mu} \nabla^{a} v\right|^{2} d x .
\end{aligned}
$$

This estimate and (82) imply that

$$
\begin{aligned}
\frac{d}{d t}\left\langle\partial_{t}^{\mu+1} \nabla^{a} v,\left[h ; \nabla \partial_{t}^{\mu} \nabla^{a} v\right]\right\rangle+\int_{\boldsymbol{R}^{d}}\left\{\frac{\left(d \phi+|x| \phi^{\prime}\right)}{2}\right\}\left|\partial_{t}^{\mu+1} \nabla^{a} v\right|^{2} d x \\
+\int_{\boldsymbol{R}^{d}}\left\{\phi+|x| \phi^{\prime}-\frac{d \phi+|x| \phi^{\prime}}{2}\right\}\left|\nabla \partial_{t}^{\mu} \nabla^{a} v\right|^{2} d x \\
\leq-\left\langle B_{\lambda} \partial_{t}^{\mu+1} \nabla^{a} v,\left[h ; \nabla \partial_{t}^{\mu} \nabla^{a} v(t)\right]\right\rangle \\
\quad-\sum_{\substack{b \leq a \\
b \neq 0}}\left(\begin{array}{l}
a \\
b
\end{array}\right)\left\langle\nabla^{b} B_{\lambda} \partial_{t}^{\mu+1} \nabla^{a-b} v,\left[h ; \nabla \partial_{t}^{\mu} \nabla^{a} v\right]\right\rangle \\
+\left\langle\partial_{t}^{\mu} \nabla^{a} N[v, v],\left[h ; \nabla \partial_{t}^{\mu} \nabla^{a} v\right]\right\rangle .
\end{aligned}
$$


Let estimate for the right side of (85). First, since $B_{\lambda}$ is a nonnegative symmetric matrix, there exists a nonnegative symmetric matrix $S_{\lambda}$ such that $S_{\lambda}^{2}=B_{\lambda}$. Using (B3) $\lambda$ and (23), for any $|a| \leq \bar{L}-\mu-1$ and $K>0$ we obtain

$$
\begin{aligned}
\left|\left\langle B_{\lambda} \partial_{t}^{\mu+1} \nabla^{a} v,\left[h ; \nabla \partial_{t}^{\mu} \nabla^{a} v\right]\right\rangle\right| \\
\quad=\left|\left\langle S_{\lambda} \partial_{t}^{\mu+1} \nabla^{a} v, S_{\lambda}\left[h ; \nabla \partial_{t}^{\mu} \nabla^{a} v\right]\right\rangle\right| \\
\quad \leq \frac{K}{4}\left\|S_{\lambda} \partial_{t}^{\mu+1} \nabla^{a} v\right\|_{2}^{2}+\frac{1}{K}\left\|S_{\lambda}\left[h ; \nabla \partial_{t}^{\mu} \nabla^{a} v\right]\right\|_{2}^{2} \\
\quad=\frac{K}{4}\left\langle\partial_{t}^{\mu+1} \nabla^{a} v, B_{\lambda} \partial_{t}^{\mu+1} \nabla^{a} v\right\rangle+\frac{1}{K}\left\langle\left[h ; \nabla \partial_{t}^{\mu} \nabla^{a} v\right], B_{\lambda}\left[h ; \nabla \partial_{t}^{\mu} \nabla^{a} v\right]\right\rangle \\
\quad \leq \frac{K}{4}\left\langle\partial_{t}^{\mu+1} \nabla^{a} v, B_{\lambda} \partial_{t}^{\mu+1} \nabla^{a} v\right\rangle+\frac{1}{K}\left\|B_{\lambda}\right\|_{\infty}\|h\|_{\infty}^{2}\left\|\nabla \partial_{t}^{\mu} \nabla^{a} v\right\|_{2}^{2} \\
\quad \leq \frac{K}{4}\left\langle\partial_{t}^{\mu+1} \nabla^{a} v, B_{\lambda} \partial_{t}^{\mu+1} \nabla^{a} v\right\rangle+\frac{\|B\|_{\infty} b_{0}^{2} R^{2}}{\lambda K}\left\|\nabla \partial_{t}^{\mu} \nabla^{a} v\right\|_{2}^{2} .
\end{aligned}
$$

So it holds that

$$
\begin{aligned}
& -\sum_{|a| \leq \bar{L}-\mu-1}\left\langle B_{\lambda} \partial_{t}^{\mu+1} \nabla^{a} v,\left[h ; \nabla \nabla^{a} \partial_{t}^{\mu} v\right]\right\rangle \\
& \quad \leq \sum_{|a| \leq \bar{L}-\mu-1} \frac{K}{4}\left\langle\partial_{t}^{\mu+1} \nabla^{a} v, B_{\lambda} \partial_{t}^{\mu+1} \nabla^{a} v\right\rangle+\frac{2\|B\|_{\infty} b_{0}^{2} R^{2}}{\lambda K} E_{\bar{L}-\mu}\left(\partial_{t}^{\mu} v\right) .
\end{aligned}
$$

Second, using (B3) $\lambda$ and (23), for $\lambda \leq 1$ we have

$$
\begin{aligned}
& \sum_{|a| \leq \bar{L}-\mu-1} \sum_{\substack{b \leq a \\
b \neq 0}}\left(\begin{array}{l}
a \\
b
\end{array}\right)\left\langle\nabla^{b} B_{\lambda} \partial_{t}^{\mu+1} \nabla^{a-b} v,\left[h ; \nabla \partial_{t}^{\mu} \nabla^{a} v\right]\right\rangle \\
& \leq \sum_{|a| \leq \bar{L}-\mu-1} \sum_{\substack{b \leq a \\
b \neq 0}}\left(\begin{array}{l}
a \\
b
\end{array}\right) \sum_{i, j, k=1}^{d}\left|\int_{\boldsymbol{R}^{d}} \nabla^{b}\left(B_{\lambda}\right)_{i j} \partial_{t}^{\mu+1} \nabla^{a-b} v^{j} h^{k} \partial_{k} \partial_{t}^{\mu} \nabla^{a} v^{i} d x\right| \\
& \leq \sum_{|a| \leq \bar{L}-\mu-1} \sum_{\substack{b \leq a \\
b \neq 0}}\left(\begin{array}{l}
a \\
b
\end{array}\right) \sum_{i, j, k=1}^{d}\left\|\nabla^{b} B_{\lambda}\right\|_{\infty}\left\|\partial_{t}^{\mu+1} \nabla^{a-b} v^{j}\right\|_{2}\left\|h^{k}\right\|_{\infty}\left\|\partial_{k} \nabla^{a} \partial_{t}^{\mu} v^{i}\right\|_{2} \\
& \leq \lambda C E_{\bar{L}-\mu}\left(\partial_{t}^{\mu} v\right) .
\end{aligned}
$$

We already got the estimate of $\left\langle\partial_{t}^{\mu} \nabla^{a} N[v, v],\left[h ; \nabla \partial_{t}^{\mu} \nabla^{a} v\right]\right\rangle$ in lemma 5.1. Combining estimates (85), (86), (87) and (70), we get (80). This completes the proof of lemma 5.3.

Proof of lemma 3.1. Let $K=C_{0} / \lambda$. Calculating $K \times(72)+b_{0}(2 d-1) /$ $4 \times(73)+(80)$ we get 


$$
\begin{aligned}
\frac{d}{d t} G_{\bar{L}, \mu}(v)+ & \sum_{|a| \leq \bar{L}-\mu-1}\left\{K\left\langle\partial_{t}^{\mu+1} \nabla^{a} v, B_{\lambda} \partial_{t}^{\mu+1} \nabla^{a} v\right\rangle-\frac{b_{0}(2 d-1)}{4}\left\|\partial_{t}^{\mu+1} \nabla^{a} v\right\|_{2}^{2}\right. \\
& \left.\quad+\int_{\boldsymbol{R}^{d}} \frac{d \phi+|x| \phi^{\prime}}{2}\left|\partial_{t}^{\mu+1} \nabla^{a} v\right|^{2} d x\right\} \\
& +\sum_{|a| \leq \bar{L}-\mu-1} \int_{\boldsymbol{R}^{d}}\left(\frac{b_{0}(2 d-1)}{4}+\phi+|x| \phi^{\prime}-\frac{d \phi+|x| \phi^{\prime}}{2}\right)\left|\nabla \partial_{t}^{\mu} \nabla^{a} v\right|^{2} d x \\
\leq & C\left(\lambda^{2} K+\frac{\lambda^{2} b_{0}(2 d-1)}{4}+\lambda\right) E_{\bar{L}-\mu}\left(\partial_{t}^{\mu} v\right) \\
& +C\left(K+\frac{b_{0}(2 d-1)}{4}+\frac{1}{\lambda}\right) D_{\bar{L}, \mu}(v) \\
& +\frac{K}{4} \sum_{|a| \leq \bar{L}-\mu-1}\left\langle\partial_{t}^{\mu+1} \nabla^{a} v, B_{\lambda} \partial_{t}^{\mu+1} \nabla^{a} v(t)\right\rangle+\frac{2\|B\|_{\infty} b_{0}^{2} R^{2}}{\lambda K} E_{\bar{L}-\mu}\left(\partial_{t}^{\mu} v\right) .
\end{aligned}
$$

From (17), it holds that

$$
r \phi^{\prime}(r)= \begin{cases}0, & (r \leq R / \lambda) \\ -\phi(r), & (r \geq R / \lambda) .\end{cases}
$$

Using (B2) $\lambda,(89), K \geq d / \lambda$ and $\phi \geq 0$, we obtain

$$
\begin{aligned}
& K\left\langle\partial_{t}^{\mu+1} \nabla^{a} v, B_{\lambda} \partial_{t}^{\mu+1} \nabla^{a} v\right\rangle-\frac{b_{0}(2 d-1)}{4}\left\|\partial_{t}^{\mu+1} \nabla^{a} v\right\|_{2}^{2} \\
& \quad+\int_{R^{d}}\left\{\frac{d \phi+|x| \phi^{\prime}}{2}\right\}\left|\partial_{t}^{\mu+1} \nabla^{a} v\right|^{2} d x \\
& \geq \frac{K}{2}\left\langle\partial_{t}^{\mu+1} \nabla^{a} v, B_{\lambda} \partial_{t}^{\mu+1} \nabla^{a} v\right\rangle \\
& \quad+\int_{|x| \leq R / \lambda}\left(-\frac{b_{0}(2 d-1)}{4}+\frac{d b_{0}}{2}\right)\left|\partial_{t}^{\mu+1} \nabla^{a} v\right|^{2} d x \\
& \quad+\int_{|x| \geq R / \lambda}\left(\frac{\lambda b_{0} K}{2}-\frac{b_{0}(2 d-1)}{4}+\frac{d-1}{2} \phi\right)\left|\partial_{t}^{\mu+1} \nabla^{a} v\right|^{2} d x \\
& \geq \frac{K}{2}\left\langle\partial_{t}^{\mu+1} \nabla^{a} v, B_{\lambda} \partial_{t}^{\mu+1} \nabla^{a} v\right\rangle+\frac{b_{0}}{4}\left\|\partial_{t}^{\mu+1} \nabla^{a} v(t)\right\|_{2}^{2}
\end{aligned}
$$

for any $|a| \leq \bar{L}-\mu-1$. Since we have 


$$
\begin{aligned}
\int_{\boldsymbol{R}^{d}}( & \left.\frac{b_{0}(2 d-1)}{4}+\phi+|x| \phi^{\prime}-\frac{d \phi+|x| \phi^{\prime}}{2}\right)\left|\nabla \partial_{t}^{\mu} \nabla^{a} v\right|^{2} d x \\
= & \int_{|x| \leq R / \lambda}\left(\frac{b_{0}(2 d-1)}{4}+b_{0}-\frac{d b_{0}}{2}\right)\left|\nabla \partial_{t}^{\mu} \nabla^{a} v\right|^{2} d x \\
& +\int_{|x| \geq R / \lambda}\left(\frac{b_{0}(2 d-1)}{4}-\frac{(d-1)}{2} \frac{b_{0} R}{\lambda|x|}\right)\left|\nabla \partial_{t}^{\mu} \nabla^{a} v\right|^{2} d x \\
\geq & \frac{3 b_{0}}{4} \int_{|x| \leq R / \lambda}\left|\nabla \partial_{t}^{\mu} \nabla^{a} v\right|^{2} d x \\
& +\int_{|x| \geq R / \lambda}\left(\frac{b_{0}(2 d-1)}{4}-\frac{b_{0}(d-1)}{2}\right)\left|\nabla \partial_{t}^{\mu} \nabla^{a} v\right|^{2} d x \\
\geq & \frac{b_{0}}{4}\left\|\nabla \partial_{t}^{\mu} \nabla^{a} v\right\|_{2}^{2} .
\end{aligned}
$$

The estimates (88), (90) and (91) imply

$$
\begin{aligned}
& \frac{d}{d t} G_{\bar{L}, \mu}(v)+\sum_{|a| \leq \bar{L}-\mu-1} \frac{K}{2}\left\langle\partial_{t}^{\mu+1} \nabla^{a} v, B_{\lambda} \partial_{t}^{\mu+1} \nabla^{a} v\right\rangle+\frac{b_{0}}{2} E_{\bar{L}-\mu}\left(\partial_{t}^{\mu} v\right) \\
& \leq C\left(\lambda^{2} K+\frac{\lambda^{2} b_{0}(2 d-1)}{4}+\lambda\right) E_{L-\mu}\left(\partial_{t}^{\mu} v\right) \\
& \quad+C\left(K+\frac{b_{0}(2 d-1)}{4}+\frac{1}{\lambda}\right) D_{\bar{L}, \mu}(v) \\
& \quad+\frac{K}{4} \sum_{|a| \leq \bar{L}-\mu-1}\left\langle\partial_{t}^{\mu+1} \nabla^{a} v, B_{\lambda} \partial_{t}^{\mu+1} \nabla^{a} v\right\rangle+\frac{2\|B\|_{\infty} b_{0}^{2} R^{2}}{\lambda K} E_{\bar{L}-\mu}\left(\partial_{t}^{\mu} v\right)
\end{aligned}
$$

Finally using $\lambda \leq 1, K=C_{0} / \lambda$ and $\left\langle\partial_{t}^{\mu+1} \nabla^{a} v(t), B_{\lambda} \partial_{t}^{\mu+1} \nabla^{a} v(t)\right\rangle \geq 0$, we get (18), which completes the proof of lemma 3.1 .

\section{Acknowledgement}

The author is deeply grateful to Mishio Kawashita for constructive comments and warm encouragement.

\section{References}

[1] Agemi, R., Global existence of nonlinear elastic waves, Invent. Math., 142 (2000), 225-250.

[2] Charao, R. C. and Ikehata, R., Decay of solutions for a semilinear system of elastic waves in an exterior domain with damping near infinity, Nonlinear Anal., 67 (2007), 398-429. 
[3] Dharmawardane, P. M. N., Nakamura, T. and Kawashima, S., Global solutions to quasilinear hyperbolic systems of viscoelasticity, Kyoto J. Math., 51 (2011), 467-483.

[4] Gilbarg, D. and Trudinger, N., Elliptic Partial Differential Equations of Second Order, 2nd ed., Grundlehren der Mathematischen Wissenschaften, 224, Springer-Verlag, Berlin, 1983.

[ 5] Ikehata, R., Fast decay of solutions for linear wave equations with dissipation localized near infinity in an exterior domain, J. Differential Equations, 188 (2003), 390-405.

[6] John, F., Blow-up for quasilinear wave equations in three space dimensions, Comm. Pure Appl. Math., 34 (1981), 29-51.

[7] Kawashima, S., Nakao, M. and Ono, K., On the decay property of solutions to the Cauchy problem of the semilinear wave equation with a dissipative term, J. Math. Soc. Japan, 47 (1995), 617-653.

[8] Klainerman, S., The null condition and global existence to nonlinear wave equations, Lectures in Appl. Math., 23, Amer. Math. Soc., Providence, RI, 1986, pp. 293-326.

[9] Matsumura, A., On the Asymptotic Behavior of Solutions of Semi-linear Wave Equations, Publ. Res. Inst. Math. Sci., 12 (1976), 169-189.

[10] Matsumura, A., Global existence and asymptotics of the solutions of the second-order quasilinear hyperbolic equations with the first-order dissipation, Publ. Res. Inst. Math. Sci., 13 (1977), 349-379.

[11] Nakao, M., Energy decay for the linear and semilinear wave equations in exterior domains with some localized dissipations, Math. Z., 238 (2001), 781-797.

[12] Nakao, M., On global smooth solutions to the initial-boundary value problem for quasilinear wave equations in exterior domains, J. Math. Soc. Japan, 55 (2003), 765-795.

[13] Racke, R., Nonhomogeneous nonlinear damped wave equations in unbounded domains, Math. Methods Appl. Sci., 13 (1990), 481-491.

[14] Sideris, T., Nonresonance and global existence of prestressed nonlinear elastic waves, Ann. of Math. (2), 151 (2000), 849-874.

[15] Tao, T., Nonlinear Dispersive equations, Local and Global Analysis, CBMS Regional Conference Series in Mathematics, 106, American Mathematical Society, Providence, RI, 2006.

[16] Taylor, M. E., Partial Differential Equations III: Nonlinear Equations, 2nd Ed., Applied Mathematical Sciences, 117, Springer, New York, 2011.

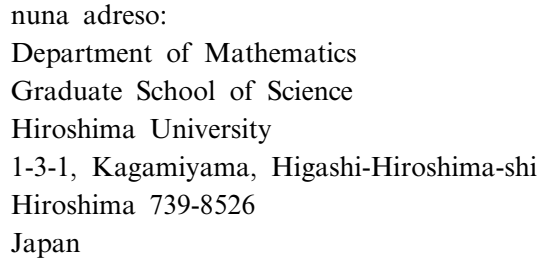

(Ricevita la 25-an de januaro, 2013)

(Reviziita la 17-an de septembro, 2014) 